Portland State University

PDXScholar

Spring 6-12-2015

\title{
Device, Method, and Algorithm to Assess Changes in Cardiac Output via Intracardiac Impedance Monitoring
}

Geoffrey Fredrick Schau

Portland State University

Follow this and additional works at: https://pdxscholar.library.pdx.edu/open_access_etds

Part of the Biomedical Commons, and the Biomedical Engineering and Bioengineering Commons Let us know how access to this document benefits you.

\section{Recommended Citation}

Schau, Geoffrey Fredrick, "Device, Method, and Algorithm to Assess Changes in Cardiac Output via Intracardiac Impedance Monitoring" (2015). Dissertations and Theses. Paper 2402.

https://doi.org/10.15760/etd.2399

This Thesis is brought to you for free and open access. It has been accepted for inclusion in Dissertations and Theses by an authorized administrator of PDXScholar. Please contact us if we can make this document more accessible: pdxscholar@pdx.edu. 
Device, Method, and Algorithm to Assess Changes in Cardiac Output

Via Intracardiac Impedance Monitoring

by

Geoffrey Fredrick Schau

A thesis submitted in partial fulfillment of the requirements for the degree of

\author{
Master of Science \\ in \\ Electrical and Computer Engineering
}

Thesis Committee:

James McNames, Chair

$\mathrm{Fu} \mathrm{Li}$

Timothy Anderson

Portland State University

2015 
(C) 2015 Geoffrey Fredrick Schau 


\begin{abstract}
Cardiac output, the volume of blood pumped by the heart over time, is a powerful clinical metric used by physicians to assess overall cardiac health and patient well-being. However, current cardiac output estimation methods are typically invasive, time-consuming, expensive, or some combination of all three. Patients that receive artificial cardiac pacemaker devices are particularly susceptible to cardiac dysfunction and often require long-term cardiac monitoring support.

This thesis proposes a novel cardiac output monitoring solution which leverages an implantable intracardiac medical device. The principles of traditional impedance cardiography, an established cardiac output monitoring technique in practice for over fifty years, have been adapted to incorporate a leadless artificial cardiac pacemaker, an implantable medical device contained entirely within the heart. This novel method, colloquially referred to as Z-Cardio, monitors time-varying intracardiac impedance modulation to assess changes in cardiac output. In this study, technologies both old and new are synthesized to produce a novel and effective method of monitoring a critical metric of cardiac health.
\end{abstract}




\section{Dedication}

To my mother for her lessons in love,

To my father for his lessons in workmanship,

To all those who came before us,

From parent to greatest of grandparent unknown,

For lighting the way. 


\section{Acknowledgements}

The work presented herein would not have been possible without the dedication, competence, and ethic of many admirable men and women. I would like to confer specific acknowledgement to Chris Moulder for providing me the opportunity and encouragement to pursue this work; Hollis Whittington, Joseph Raven, Randy Armstrong, and Gregory Timmel for their facilitation of the data collection studies that supported this study; Andrew Kibler, Min Qu, Chris de Voir, and Jie Lian for their professional expertise, guidance, and companionship; Iris Shelly for her encouragement and unparalleled eye for detail; and my academic adviser, James McNames, for his unwavering support for his students, dedication to the engineering craft, depth of knowledge, profound patience, leadership in forging a better learning experience, and whose work ethic will forever remain an inspiration.

My personal relationships with these men and women have left me deeply humbled. It is with utmost sincerity that I thank each and every one of them for contributing to my personal and professional development, for incubating my interests and ideas, for entertaining my inquiries, and who, through their work, make our world a better place to live. 


\section{Table of Contents}

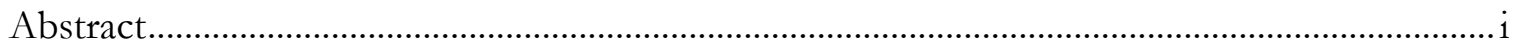

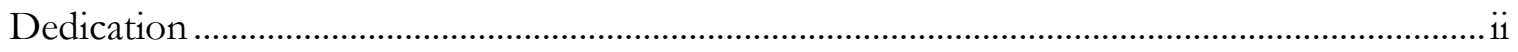

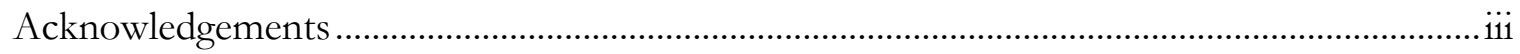

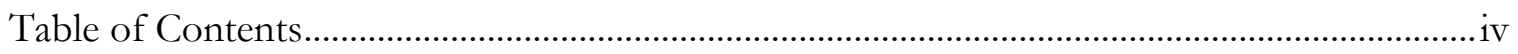

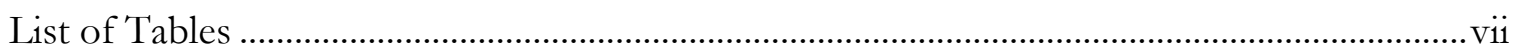

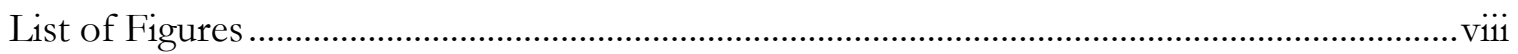

List of Abbreviations and Acronyms ......................................................................................

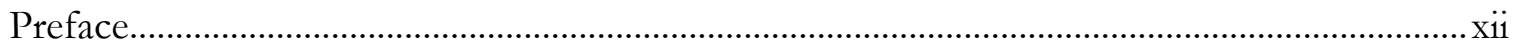

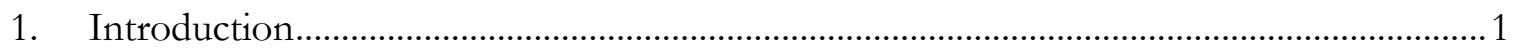

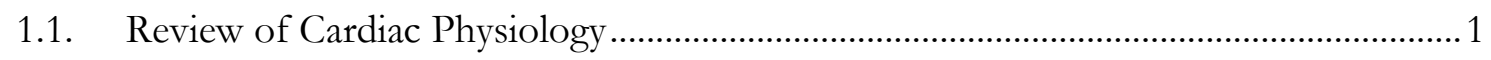

1.1.1. The Heart as a Mechanical System ...................................................................... 2

1.1.2. The Heart as an Electrical System ................................................................ 4

1.1.3. The Heart as a Chemical System ……..............................................................

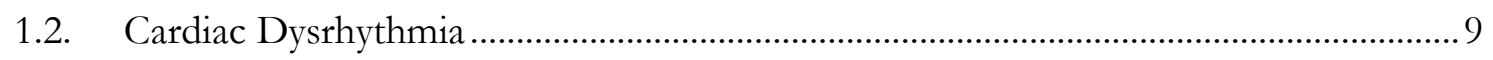

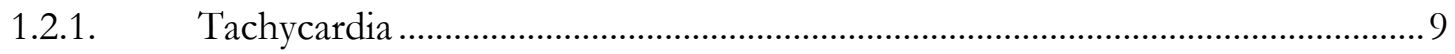

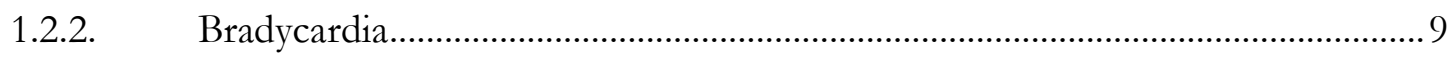

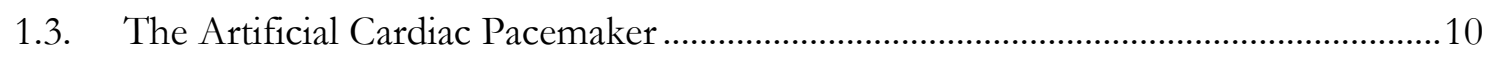

1.3.1. Injectable Leadless Pacemaker ........................................................................... 11

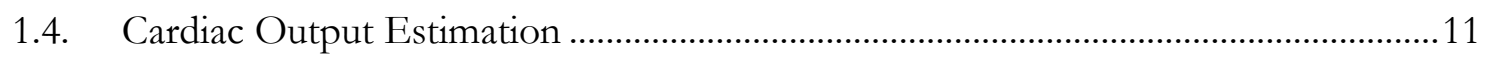

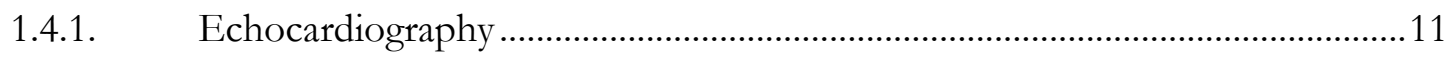

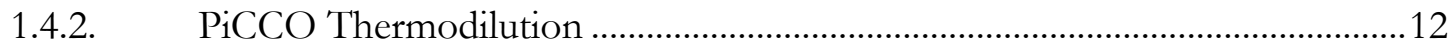

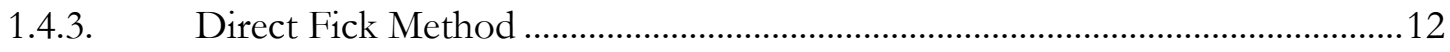




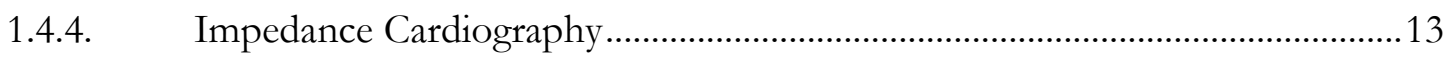

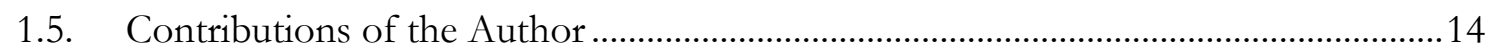

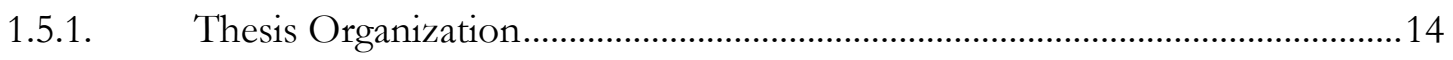

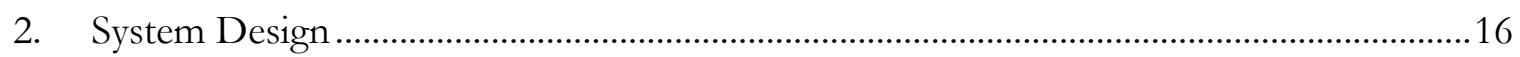

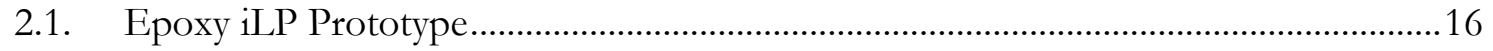

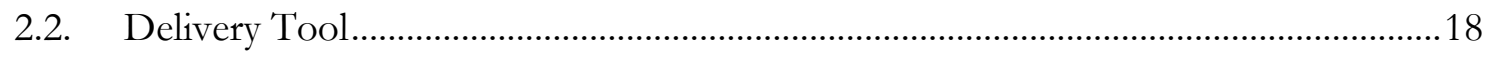

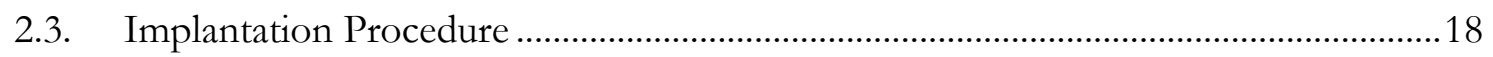

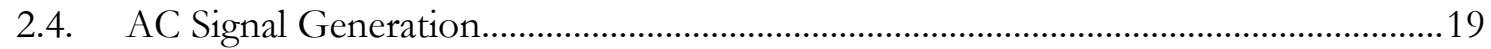

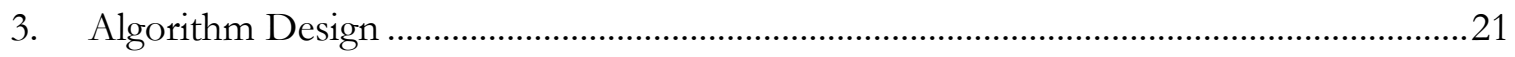

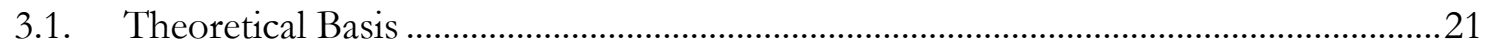

3.2. Mathematical Model of Expected Impedance Changes................................................23

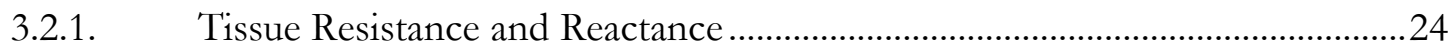

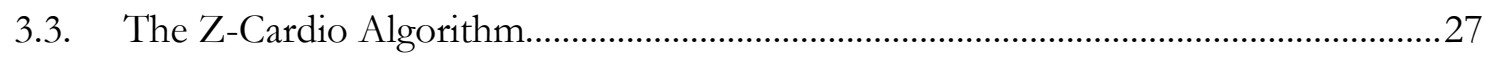

4. Simulator Design Requirements and Verification.................................................................33

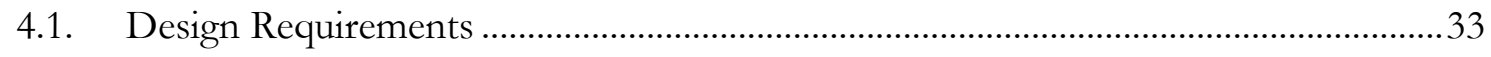

4.1.1. Low-Cost and Rapid-Development Design...................................................... 33

4.1.2. Controllable and Measurable Simulator Stroke Volume .....................................33

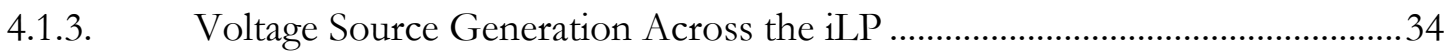

4.1.4. Blood and Myocardial Tissue Emulation ............................................................ 34

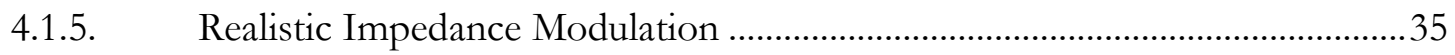

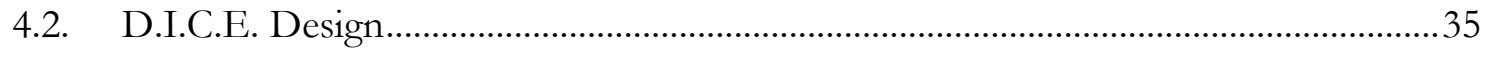

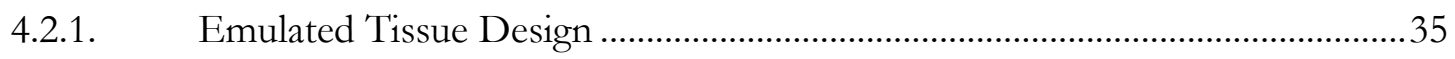

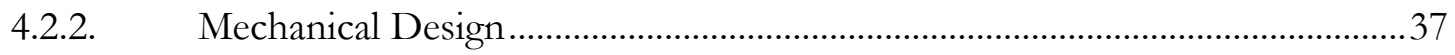




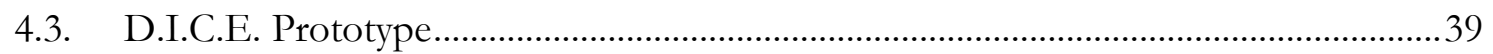

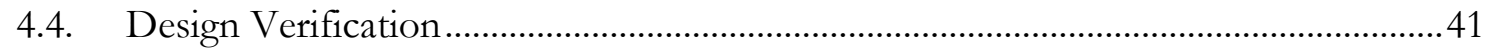

5. Simulator Analysis, Results, and Discussion....................................................................... 44

5.1. Algorithm Performance and Stroke Volume Comparison .........................................4 44

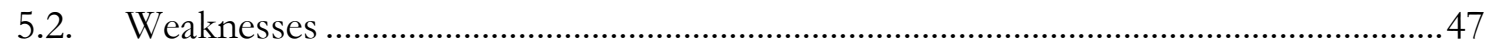

6. Animal Study Design, Results, and Discussion .................................................................. 49

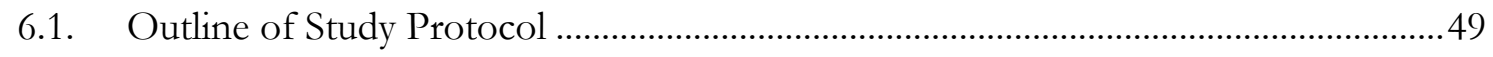

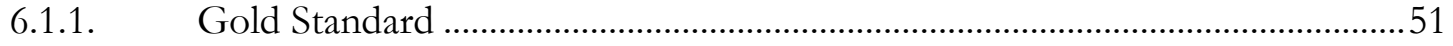

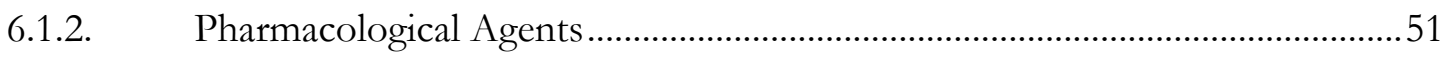

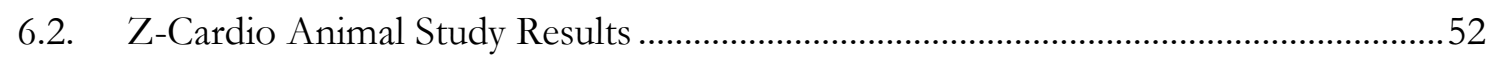

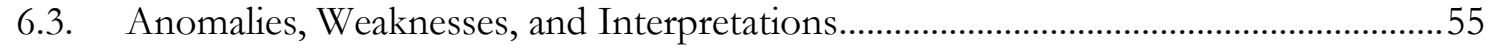

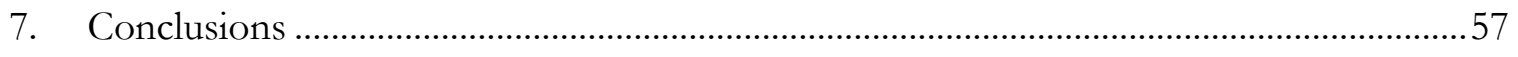

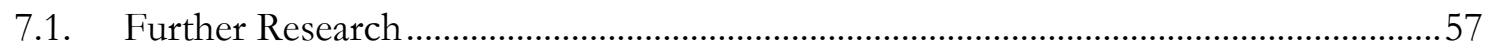

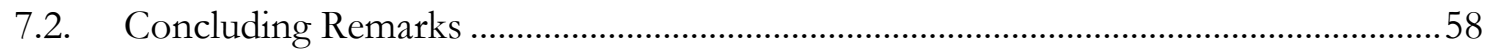

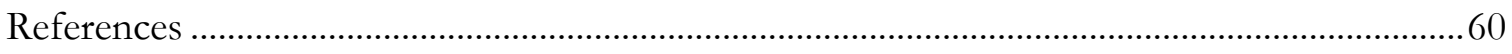

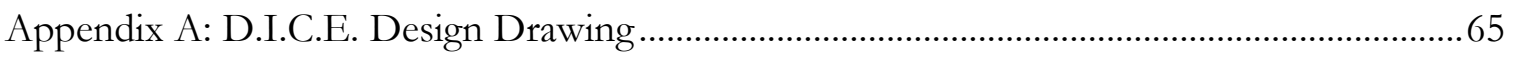

Appendix B: In Vivo Data Collection Study Protocol ................................................................66

Justification of Testing System and Animal Arrangements ...................................................66

Justification of Test Hardware Design ..................................................................................69

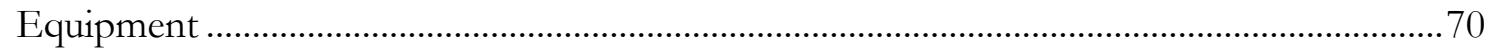

Appendix C: Z-Cardio Data Collection Procedure ..................................................................... 71 


\section{List of Tables}

Table 1: Parameters utilized in the nested cylinder mathematical model ..................................24

Table 2: Pharmacological drugs administered as part of this study ............................................69

Table 3: Quantity and equipment employed throughout the preliminary data collection study 


\section{List of Figures}

Figure 1: Basic cardiac anatomy. Blue components indicate de-oxygenated blood pathways and red components indicate oxygenated blood pathways (Wikimedia Commons ZooFari/CC BY-SA 3.0)

Figure 2: Electrical wiring diagram of the heart (Wikimedia Commons Angelito7/CC BY-SA

Figure 3: A Wiggers diagram, showing the cardiac cycle events occurring in the left ventricle (Wikimedia Commons xavax/CC BY-CA 2.5)

Figure 4: Cardiac pacemaker cell membrane potential (Wikimedia Commons OpenStax

College/CC BY-SA 3.0)

Figure 5: Cardiomyocyte cell membrane potential (Wikimedia Commons OpenStax

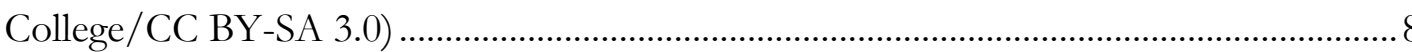

Figure 12: Diagram of iLP and connecting terminal.................................................................17

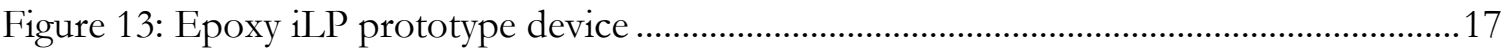

Figure 14: Approximate locations of signal injection electrodes indicated by grey circles.

Grey circles indicate approximate current injection electrodes. iLP shown to scale ......20

Figure 15: Single cardiac cycle visualized both with the intracardiac impedance curve and the

IEGM

Figure 16: Simplified model of the right ventricle used to mathematically predict expected impedance changes

Figure 17: Mathematical prediction of resistances for blood, myocardium, and their functional equivalent .26

viii 
Figure 18: Model circuit diagram of the body where elements specific to the heart are circled in red .27

Figure 19: High-level flow diagram of the Z-Cardio algorithm 28

Figure 20: Illustration of the Z-Cardio algorithm processing cascade. The top plot shows the raw data in black and the low-pass trend in yellow. The middle plot shows the differential of the yellow trace from the top plot, and highlights the positive portion (half-wave rectification) of the curve in red. The bottom plot shows the integral of the red curve shown in the middle plot for each beat.

Figure 21: Resistivity of blood and myocardium over the nominal voltage generation frequency band

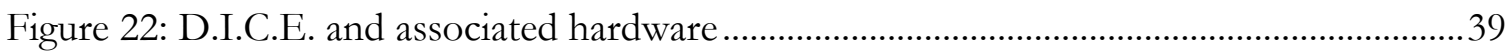

Figure 23: Close-up photographs of the D.I.C.E. simulator

Figure 24: Simulator contraction dynamics of increasing stroke volumes indicated in the graduated cylinder by black arrows.

Figure 25: Regression analysis between D.I.C.E. stroke volume and impedance across the iLP42

Figure 26: Filtered intracardiac impedance signal collected from an iLP embedded in the D.I.C.E

Figure 27: Illustration of the Z-Cardio algorithm signal processing cascade applied to simulated data .46

Figure 28: Linear fit between Z-Cardio stroke volume indices and the measured stroke volume of the D.I.C.E. system. The blue dots are individual measurements while the red dots are the average for the measurements made for each stroke volume 
Figure 29: Stroke volume changes measured over time by both the PiCCO monitor (left) and

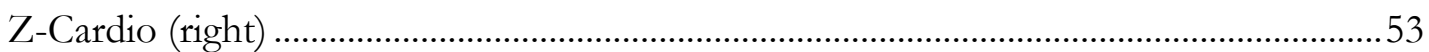

Figure 30: Cardiac output changes measured over time by both the PiCCO monitor (left)

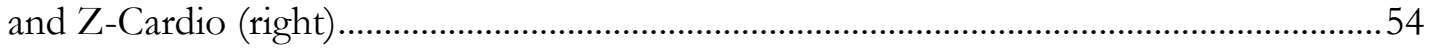

Figure 31: Regression analysis of cardiac output estimations by PiCCO and Z-Cardio ..........55 
List of Abbreviations and Acronyms

\begin{tabular}{ll}
\hline Abbreviation & Definition \\
\hline CO & Cardiac Output \\
CVP & Central Venous Pressure \\
DFT & Drawn Filled Tube \\
ECG & Electrocardiogram \\
HR & Heart Rate \\
IACUC & Institutional Animal Care and Use Committee \\
ICG & Impedance Cardiography \\
IEGM & Intracardiac Electrogram \\
iLP & Injectable Leadless Pacemaker \\
IVC & Inferior Vena Cava \\
LA & Left Atrium \\
LV & Left Ventricle \\
NIDAQ & National Instruments Data Acquisition System \\
PBS & Phosphate-Buffered Saline \\
PVC & Premature Ventricular Contraction \\
RA & Right Atrium \\
RV & Right Ventricle \\
SA & Sinoatrial \\
SOP & Standard Operating Procedure \\
SV & Stroke Volume \\
SVC & Superior Vena Cava \\
VET & Ventricular Ejection Time \\
\hline & \\
\hline
\end{tabular}




\section{Preface}

The purpose of this thesis is to clearly and concisely describe a system designed to monitor changes in cardiac output, the volume of blood that flows through the heart. This thesis would not have been possible without a sound understanding of both electrical and mechanical principles that govern our heart's function. Living systems balance remarkably intricate components in the desperate struggle for mere temporary survival. Despite the complexity and variability of the interconnecting systems within even a single organism, few are as intimately tied to our own human sense of well-being as our own beating heart. Over an average lifetime, the human heart will contract nearly four billion times, pumping approximately one quarter of a billion liters of blood throughout a single body. Unwavering in consistency, the ever-present beating within our chests has assured even our earliest human ancestors of their own vitality and liveliness. Incredibly, for the vast majority of us, our hearts pump virtually nonstop and without failure for all but the absolute final moments of existence.

Unfortunately, such a system is not without fault. For many reasons, the heart can and does break down. If a heart becomes incapable of supplying a body with oxygen-rich blood, the body's carefully maintained metabolic balance may begin to collapse. Understanding whether our hearts are pumping enough blood is the principle motivation for the investigation that I have undertaken. The objective of this thesis is to build upon existing technology and to establish a new method of cardiac performance assessment. Through this, I seek to provide greater insight into the intimate and incredible organ that we all share. 


\section{Introduction}

Cardiac output measures the rate of blood flow through the heart by relating the volume of blood ejected from the heart in a single contraction and the rate at which the heart contracts. For a typical healthy man and women, nominal cardiac output is approximately 5.6 and 4.9 liters per minute, respectively [1]. While a healthy heart is adequately capable of supplying oxygen-rich blood to the body, a decrease in cardiac output over time may indicate heart failure, necessitating therapeutic intervention such as implanting an artificial cardiac pacemaker [2]. Doctors interested in monitoring their patients' cardiac output are faced with a variety of methods to choose from. Patients who have already received an artificial cardiac pacemaker would benefit from a device capable of accurately monitoring changes in cardiac output over time, providing physicians a powerful tool in developing treatment options for their patients [3]. This thesis explores a novel method by which an implanted medical device may assess changes in cardiac output by monitoring changes in the electrical impedance across an electric dipole located within the right ventricle. To better facilitate the reader's understanding of the principles described in this thesis, a background in human cardiac physiology, the artificial cardiac pacemaker, and the relevant biophysical principles is provided.

\subsection{Review of Cardiac Physiology}

The mammalian circulatory system is driven by the heart, the body's principal pump of both oxygen and nutrients throughout the body. This section will introduce the high-level principles of cardiac operation by discussing the mechanical, electrical, and chemical behavior of the human heart.

This thesis is concerned with three primary cardiac metrics: heart rate, stroke volume, 
and cardiac output. Heart rate (HR) is the rate at which the heart contracts. Heart rate is typically measured in beats per minute and can vary significantly between individuals but typically rests around 70 beats per minute [4]. The heart's stroke volume (SV), typically measured in milliliters, is the volume of blood ejected from the heart in a single heartbeat, approximately 70 milliliters in a healthy human male. Cardiac output (CO) is the total volume of blood pumped through the heart over unit time, typically measured in liters per minute, and rests at approximately 5 liters per minute in a healthy human male. The following sections will discuss the physiology governing these metrics, challenges associated when any of these metrics begins to collapse, and treatment options available to provide cardiac output monitoring support. This chapter concludes with a brief discussion of contributions made by the author and outlines the organization of the rest of this thesis.

\subsubsection{The Heart as a Mechanical System}

The human heart is divided into four pumping chambers [5]. The cyclical and coordinated contraction of the four chambers is referred to as the cardiac cycle. The cardiac cycle is divided into two distinct phases. The diastolic phase, or diastole, refers to time during which the heart is relaxing; the systolic phase, or systole, refers to time during which the heart is contracting and ejecting blood. Following the flow of blood through the heart in Figure 1, deoxygenated venous blood first returns from the body to the heart through the superior vena cava (SVC) and the inferior vena cava (IVC), which are located above and below the heart, respectively. These two great veins draw blood from everywhere else in the body and deliver blood to the heart's right atrium (RA). The RA contracts to deliver blood through the tricuspid valve to prime the right ventricle (RV) for contraction. The right ventricle pumps blood through the pulmonary valve, through the pulmonary arteries, and into the oxygen-supplying 
lungs. Newly oxygenated blood returns to the heart from the lungs through the pulmonary veins, which join at the left atrium (LA). The LA pumps oxygen-rich blood through the mitral valve and into the left ventricle (LV), priming the largest and most powerful chamber of the heart to efficiently deliver blood throughout the rest of the body. As the muscular left ventricle contracts, blood is forced out of the heart through the aortic valve, through the aorta, and out to the body's systemic tissue. Through this cycle, energy is transferred to the blood, providing enough velocity and pressure to reach systemic tissue within the body to deliver oxygen and nutrients, fulfillinh metabolic demand.

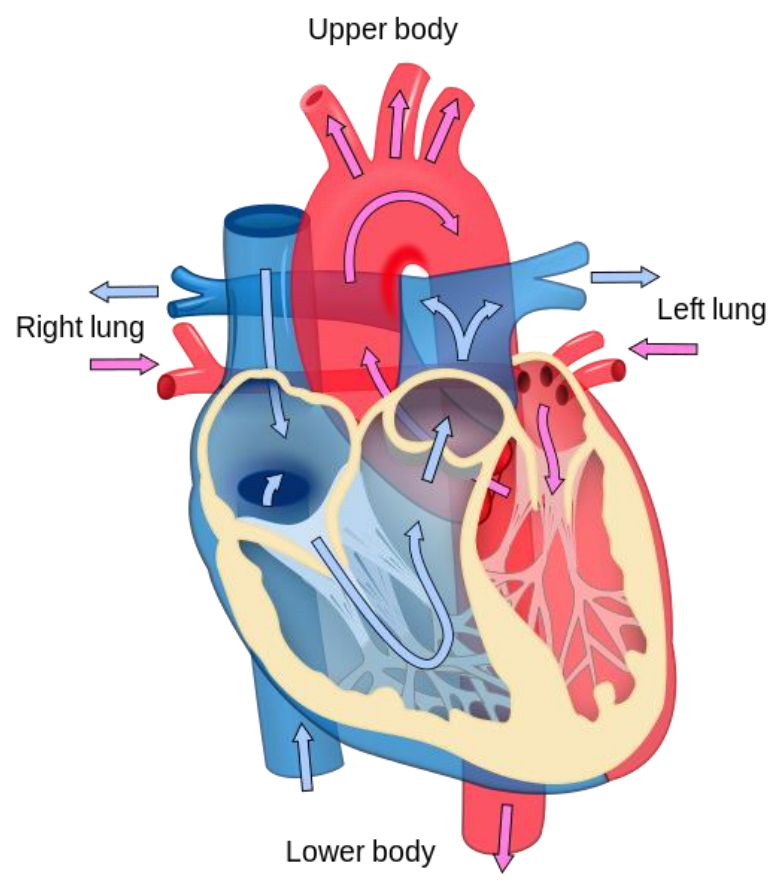

Figure 1: Basic cardiac anatomy. Blue components indicate de-oxygenated blood pathways and red components indicate oxygenated blood pathways (Wikimedia Commons ZooFari/CC BY-SA 3.0)

The heart requires its own supply of oxygenated blood to function properly. The coronary vascular system supplies the heart with its own blood by drawing oxygenated blood directly from the aorta, diffusing oxygen through coronary vasculature, and expelling deoxygenated blood directly into the right atrium through the coronary sinus. A myocardial 
infarction, commonly referred to as a heart attack, typically describes a sudden blockage within a coronary artery, which may result in cellular necrosis or complete cardiac arrest [6].

\subsubsection{The Heart as an Electrical System}

The heart is controlled by both extrinsic and intrinsic electrical activity. The autonomic nervous system controls the heart's rate of contraction (chronotropy), neural conduction velocity (dromotropy), and myocardial tissue contractility (inotropy). Electrical impulses travel from the brain and down the Vagus nerve, which innervates the heart with both sympathetic and parasympathetic neural fibers. Together, the competing sympathetic and parasympathetic systems control and modulate cardiac neural tone [7].

The heart's rhythmic cardiac cycle is controlled by electrical impulses originating from the sinoatrial (SA) node within the heart. The SA impulse propagates around the right and then left atrium, inducing atrial contraction, before rejoining at the atrioventricular (AV) node, located between the right atrium and right ventricle. From the AV node, the impulse travels downward through the Bundle of His, a dense neural structure that runs through the center of the ventricular septum. As the impulse reaches the apex of the heart, it branches into the left and right bundle branches before reversing direction, travelling back up through the outer walls of both the right and the left ventricle. The left and right bundles splinter into Purkinje Fibers, which fully innervate both the left and right ventricles. As electrical signals induce contraction in this way, a controllable, predictable, and synchronized cardiac motion results. A detailed wiring diagram of the heart is shown below in Figure 2. 


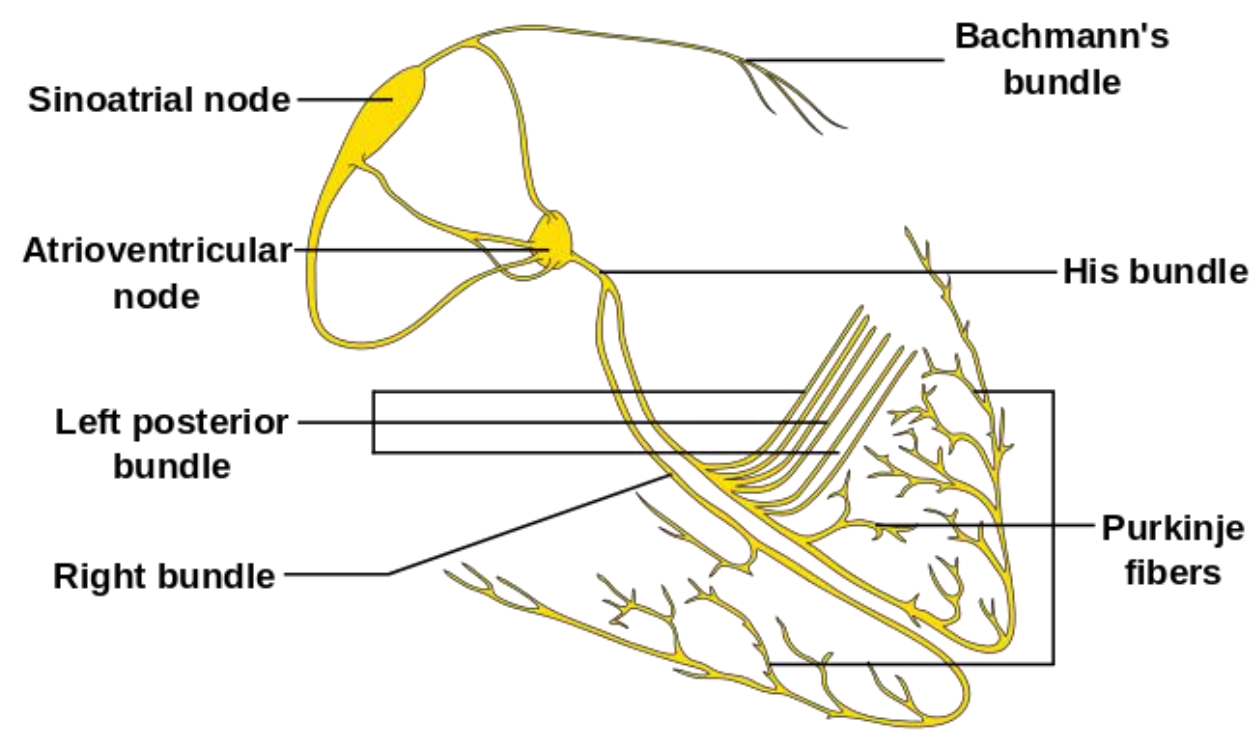

Figure 2: Electrical wiring diagram of the heart (Wikimedia Commons Angelito7/CC BY-SA 3.0)

The electrical activity of the heart can be detected by surface electrodes on the skin through the electrocardiogram (ECG) [8]. An example ECG with associated mechanical behavior of the heart, known as a Wiggers Diagram, is shown below in Figure 3. One of the most ubiquitous diagnostic tools in medicine, the ECG visualizes the heart's electrical behavior. Typically, the ECG is divided into 3 distinct segments: the P wave, corresponding to atrial contraction; the QRS complex, corresponding to synchronized ventricular contraction and atrial repolarization; and the $\mathrm{T}$ wave, corresponding to synchronized ventricular repolarization [9]. 


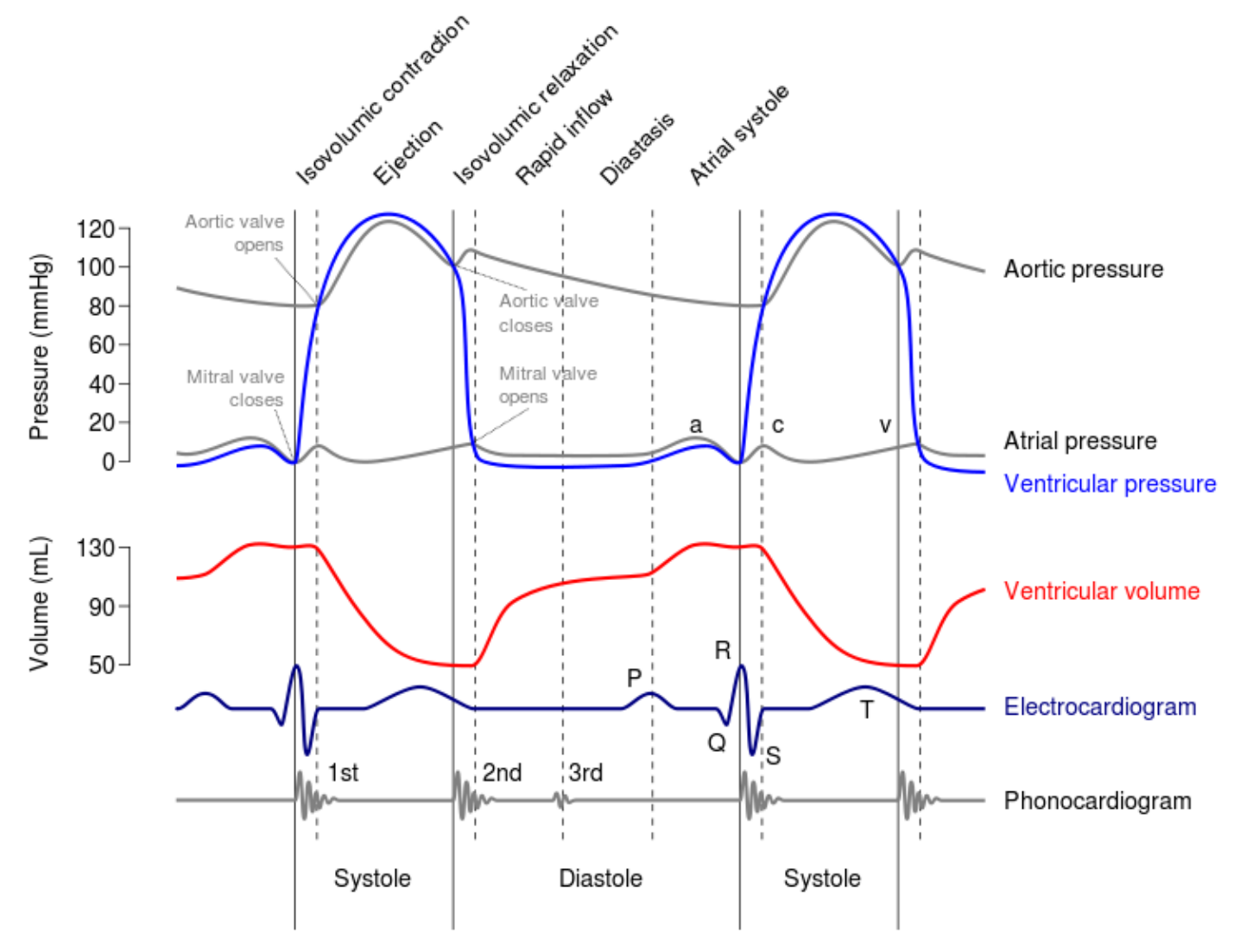

Figure 3: A Wiggers diagram, showing the cardiac cycle events occurring in the left ventricle (Wikimedia Commons xavax/CC BY-CA 2.5)

The ECG is electromechanically coupled to the myocardium [10]. Under normal operating conditions, electrical signals on an ECG are followed by mechanical contraction after some electomechanical delay. Contraction is evidenced by cyclic changes in both pressure and volume within the heart, which are also illustrated above. The phonocardiogram, commonly measured with a stethoscope, monitors heart sounds associated with the closure valves within the heart.

As the electrical wave-front propagates through the heart, the acting membrane potential of the cardiac conduction cells induce biochemical action, resulting in mechanical contraction of cardiac cells. The timing of the heart's electrical system corresponds to the 
chemically-based contraction of the entire organ.

\subsubsection{The Heart as a Chemical System}

Cells within the heart are divided into two classes: cardiomyocytes and pacemaker cells. These cells make up approximately $99 \%$ and 1\% of all heart cells, respectively [11]. Cardiac cells are autorythmic, meaning they will spontaneously contract at a fixed rate without external stimulation if left unstimulated.

Cardiac autorhythmicity results from a unique property of cardiac pacemaker cells where they, unlike other muscle or neuron cells, depolarize themselves. This behavior results from unique "leaky" sodium ion channels, which slowly permit sodium influx into the cell, passively decreasing transmembrane potential until voltage-gated calcium channels open and fully depolarize the cell. An example of the three-stage pacemaker cell depolarization process is shown below in Figure 4.

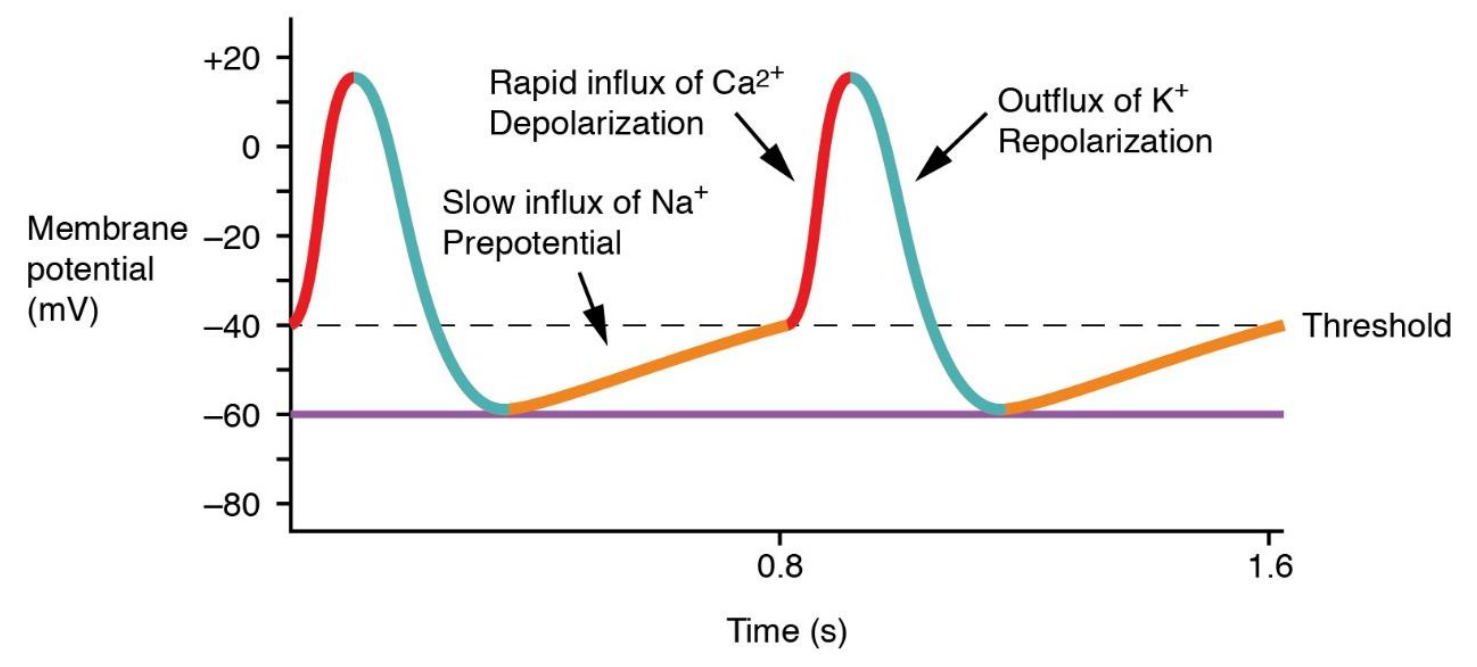

Figure 4: Cardiac pacemaker cell membrane potential (Wikimedia Commons OpenStax College/CC BY-SA 3.0)

Cardiac pacemaker cells, and in particular those located in the SA node, are responsible for the rhythmic stimulation and resulting contraction of the myocardium. Pacemaker cells 
stimulate the remaining cardiomyocytes by transmitting the self-generated transmembrane potential through the rest of the organ, opening voltage-gated ion channels that affect internal cellular organelles. An example of the cardiomyocyte membrane potential is shown below in Figure 5.

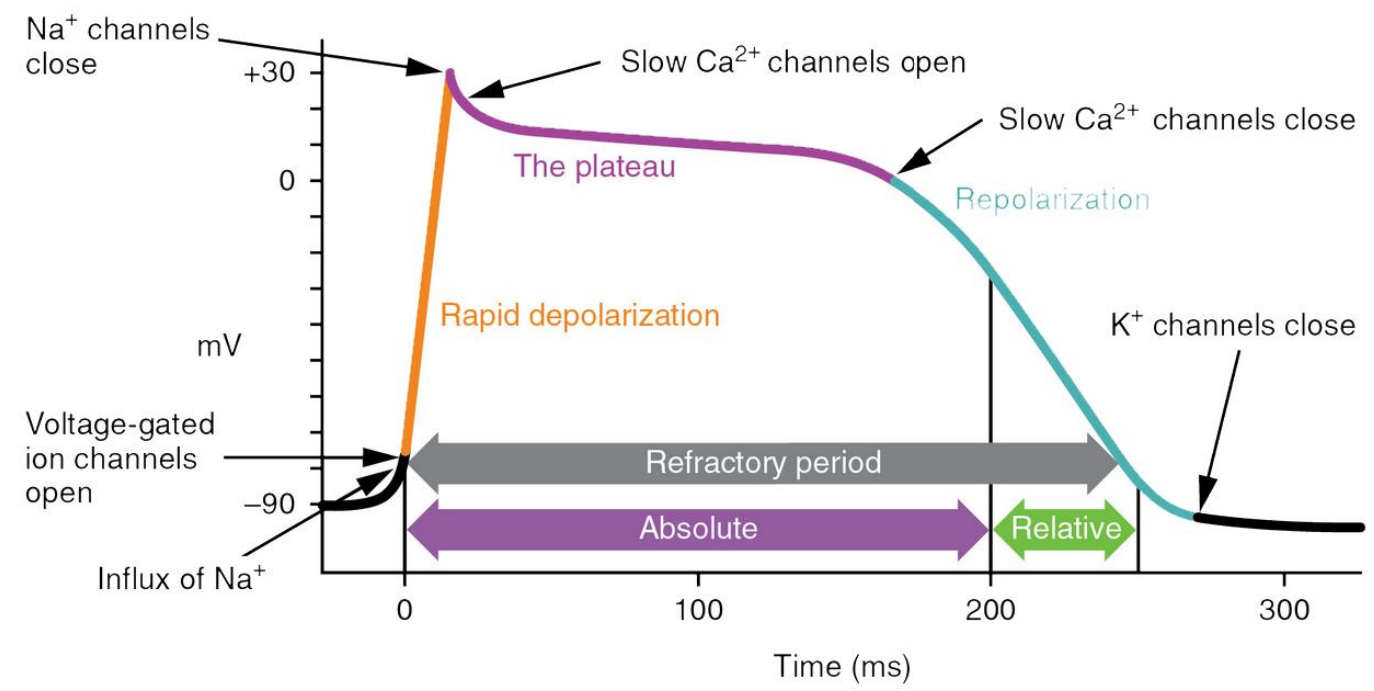

Figure 5: Cardiomyocyte cell membrane potential (Wikimedia Commons OpenStax College/CC BY-SA 3.0)

The opening of calcium ion channels by the pacemaker potential induces a chemical change within the myofibril, a specialized organelle structure present in most muscle cells throughout the body. Myofibrils contain long chains of sarcomeres, which, as the fundamental contractile unit muscle, contract in the presence of calcium ions through a complex biochemical cascade. When coupled with thousands of other cardiomyocytes, this small effect in a single cell results in the macroscopic contraction dynamics of the heart [12].

The mechanical, electrical, and chemical subsystem of cardiac function completely characterize the heart's behavior. Each connected subsystem is dependent on the other two for metabolic support; therefore, any failure within one system will cause fault in the other two. When the whole organ begins to fail in this way, cardiac dysrhythmia results. 


\subsection{Cardiac Dysrhythmia}

A cardiac dysrhythmia, or arrhythmia, describes any group of conditions where electrical activity of the heart is either too slow or too fast but that either ways results in a nonoptimal supply of blood to the body [13]. Cardiac dysrhythmias are typically classified as either tachycardia or bradycardia depending on the nature of the electrical failure within the heart.

\subsubsection{Tachycardia}

Tachycardia is a condition of high resting heart rate or irregular periods of high ventricular rate (HVR) [14]. A patient experiencing tachycardia is at risk for ventricular fibrillation (VF), an often fatal event during which the heart loses any sense of synchrony and ceases to effectively pump blood through the body. The heart may be resuscitated by a large voltage pulse delivered either internally or externally by a defibrillator [15]. To defibrillate a heart, a device, either external to or internal to the patient, generates a large voltage potential across the heart, inducing complete and instantaneous activation of myocardial voltage-gated ion channels. Following a complete reversion, leaky ion channels reestablish natural intrinsic rate. Although painful, a powerful shock delivered in time is often enough to terminate the fibrillation episode and resuscitate the patient.

\subsubsection{Bradycardia}

Unlike tachycardia, bradycardia is characterized by an abnormally slow resting heart rate typically below 60 beats per minute [16]. Greater duration between beats results in a decreased cardiac output, which could result in feelings of faintness, dizziness, or tiredness. Surface ECG recordings of bradycardia appear similar to a healthy ECG except for greater duration in the T-P interval 
Possible causes and available treatment options for cardiac dysrhythmia are diverse. One common treatment option available to patients is the implantation of an artificial cardiac pacemaker. An artificial cardiac pacemaker is an implantable device designed to improve the electrical stimulation and timing of the heart by interfacing directly with myocardial tissue. A pacemaker stimulates the heart without need for the natural electrical control system, thereby providing a reliable means of maintaining sufficient contraction dynamics to fulfill metabolic demand.

\subsection{The Artificial Cardiac Pacemaker}

An artificial cardiac pacemaker is an implantable medical device designed to alleviate symptoms associated with cardiac dysrhythmia by interfacing with the heart's natural electrical conduction system, permitting the device to sense intrinsic electrical activity and to administer artificial electrical pacing pulses as needed. Bradycardia can be treated by a typical pacemaker capable of supplying appropriately timed current pulses at a healthy rate.

A functional pacemaker is composed of two components: the can and the lead. The can is a titanium-encased module containing circuitry, battery, and, in the case of defibrillators, a large charge-storing capacitor. The lead is a long, flexible, insulated wire that connects the can to the heart tissue. Because pacemaker cans are large, they are typically implanted in a pocket under the skin somewhere superficial to the pectoral muscle. While pacemaker technology has been available for several decades, recent developments may revolutionize device design through miniaturization of the pacemaker can such that the entire device may be implanted directly in the heart without necessitating a lead. 


\subsubsection{Injectable Leadless Pacemaker}

Pacemaker technology developments have been principally focused on miniaturizing and improving device longevity. Recent technological advancements in battery storage and hardware miniaturization equip researchers with the possibility of removing the pacemaker lead from the system and collapsing the entire design into a device small enough to permanently reside within the right ventricle. Ideally, a leadless pacemaker would deliver electric charge near the apex, or bottom tip, of the right ventricle to promote even depolarization of cardiac tissue across both the right and left ventricles.

Breaking away from nearly 50 years of pacemaker design precedent, removing the pacing lead from the design promises to absolve the device of a significant weakness and promote greater patient well-being [17]. Patients who require an artificial cardiac pacemaker often require long-term cardiac output monitoring. Therefore, an artificial cardiac pacemaker capable of estimating cardiac output is desired.

\subsection{Cardiac Output Estimation}

Physicians have many options to estimate and monitor a patient's cardiac output. However, current methods require invasive catheterization, bulky external machinery, and/or highly trained medical specialists. Typical applications for cardiac output monitoring are divided into two classes: continuous and discrete monitoring, differentiated by the rate at which cardiac output values are reported. The following sections describe a few modern best practices commonly used to assess patient cardiac output.

\subsubsection{Echocardiography}

Echocardiography estimates cardiac output, stroke volume, and heart geometry 
through 2-D and 3-D Doppler shift analysis to produce images of the heart. While effective, echocardiography requires both expensive machinery and a trained echocardiologist to operate effectively, as determined by the American Society of Echocardiography. Although widely recognized as a gold standard of continuous cardiac output monitoring, costs associated with echocardiography can be prohibitively high, routinely costing up to $\$ 2000$ per session, often rendering use of echocardiography infeasible for use in developing nations ${ }^{1}$ [18].

\subsubsection{PiCCO Thermodilution}

Pulse index Continuous Cardiac Output (PiCCO) monitoring leverages thermodilution principles to assess changes in rate of blood flow through the heart. PiCCO requires a thermistor and pressure catheter placed in or near the femoral artery to measure continuous changes in arterial pulse pressure [19], making it a more invasive solution than other less-invasive options. Pulse pressure contours is calibrated through cold saline bolus injections where the rate at which the cold saline dilutes is dependent on the amount of blood flowing through the heart.

\subsubsection{Direct Fick Method}

The Direct Fick Method leverages assumptions made by the Fick Principle that relates cardiac output to the rate of gas exchange in systemic tissue [20]. Because the Direct Fick Method requires drawing blood and gas content analysis, cardiac output estimations take place at long intervals, which makes the Direct Fick Method a poor choice for continuous cardiac output monitoring.

\footnotetext{
${ }^{1}$ Or by hungry graduate students.
} 


\subsubsection{Impedance Cardiography}

Impedance cardiography (ICG) measures transthoracic impedance to assess estimations of stroke volume, cardiac output, and vascular resistance [21]. ICG requires two pairs of external transcutaneous leads; the first pair transmits a sinusoidal current through the thoracic cavity while the second pair measures the resultant voltage. Current is typically injected at $100 \mathrm{kHz}$ and $4 \mathrm{~mA}$ to achieve optimal signal propagation without inducing cellular contraction [22]. The measured voltage is dependent on thoracic impedance. As the heart transfers fluid through the great vessels, thoracic impedance changes throughout the cardiac cycle.

The electrical impedance between two leads is denoted with the letter Z. Ventricular ejection time (VET) is the period during when both pulmonary and aortic heart valves are open and blood is leaving the heart. Stroke volume is estimated with the intracardiac impedance waveform by the following equation.

$$
\text { Stroke Volume } \propto \int_{V E T} \frac{d Z}{d t}
$$

By integrating the rate at which impedance changes during the cardiac cycle, a stroke volume index measurement is made. The reliability of impedance cardiography has been subject to debate, particularly when measuring low cardiac output [23]. Challenges associated with variable body types, placement of intracardiac shunts, or valvular regurgitation contribute to measurement distortion. However, impedance cardiography benefits from high reproducibility and an ability to resolve small changes in cardiac output on a frequent and ongoing basis making it a desirable, though challenged, method of cardiac output monitoring. 


\subsection{Contributions of the Author}

The purpose of this thesis is to introduce a novel means of monitoring changes in cardiac output. Like impedance cardiography, the proposed method illustrated in this thesis applies an alternating signal across the thorax; however, unlike traditional impedance cardiography where the impedance signal is measured from the surface of the skin, the proposed method monitors changes in sensed voltage from directly within the heart itself. This method may provide a more reliable measurement of stroke volume because it may not be as sensitive to other thoracic volume conductors, such as the great vessels or lungs, which may contribute to measurement distortion expected in traditional impedance cardiography. To process the intracardiac impedance signal and estimate changes in cardiac output, a novel algorithm has been developed.

To illustrate the feasibility of the proposed method, the following steps were taken. First, a mathematical model of the heart was developed to predict expected changes observed from an intracardiac device located in the heart. Second, a mechanical simulator, designed and built to replicate expected changes in intracardiac impedance, was used to test and improve the preliminary algorithm prototype. Lastly, the method and algorithm were validated in a controlled large animal study. Results obtained from these studies, a discussion of their implications, conclusions drawn from this work, and potential future avenues of investigation are provided.

\subsubsection{Thesis Organization}

This thesis is organized into seven chapters. This first chapter has provided an introductory review of cardiac physiology, artificial cardiac pacemaker technology, and current 
best practices of monitoring cardiac output. The second chapter describes the hardware system employed throughout this thesis, including a prototype implantable device, delivery tools, and implantation procedure. The third chapter describes a novel algorithm designed to monitor changes in cardiac output. The fourth chapter introduces a mechanical simulator designed and built to replicate cardiac dynamics expected within a living subject used to demonstrate feasibility of the algorithm described in chapter three. The fifth chapter discusses results obtained from the simulator and a brief discussion of how the results contribute to system validation. The sixth chapter discusses results obtained from an in vivo animal study and provides a discussion of both the value and limitations of the results. Lastly, the seventh and final chapter summarizes conclusions drawn from this work and provides recommendations for further avenues of research. 


\section{System Design}

The following chapter describes the system employed in this thesis to monitor changes in cardiac output. This chapter discusses the prototype intracardiac device, the requisite delivery tools, and the clinical procedures for delivering a mechanical prototype into the heart. These components were developed by Micro Systems Engineering, Inc. and were repurposed by the author for this investigation.

\subsection{Epoxy iLP Prototype}

In exploring leadless pacemaker performance, injectable leadless pacemaker (iLP) device prototypes were developed. The mechanical size and distance between electrodes of the epoxy device were analogous to the size and shape of a fully functional iLP device. This surrogate device does not contain any embedded hardware and was developed to record data from outside of the heart. The prototype device is composed of two electrodes: one at the rear of the device (the hitch) and one at the screw, which anchors the device into the myocardium. The two electrodes are separated by a fixed distance of $35 \mathrm{~mm}$. The body of the device is molded from Loctite M-31CL Hysol, a medical-grade and non-conductive epoxy. The two electrodes are connected to drawn-filled tube (DFT) wires, connected to the iLP electrodes at one end and an industry standard IS-1 pacemaker lead connector on the other. When implanted, the DFT wires travel from the device out of the body where the IS-1 connector remains available for data collection. A simple diagram of the device is shown below in Figure 6 illustrating how each of the iLP electrodes is connected to an external IS-1 connector. 


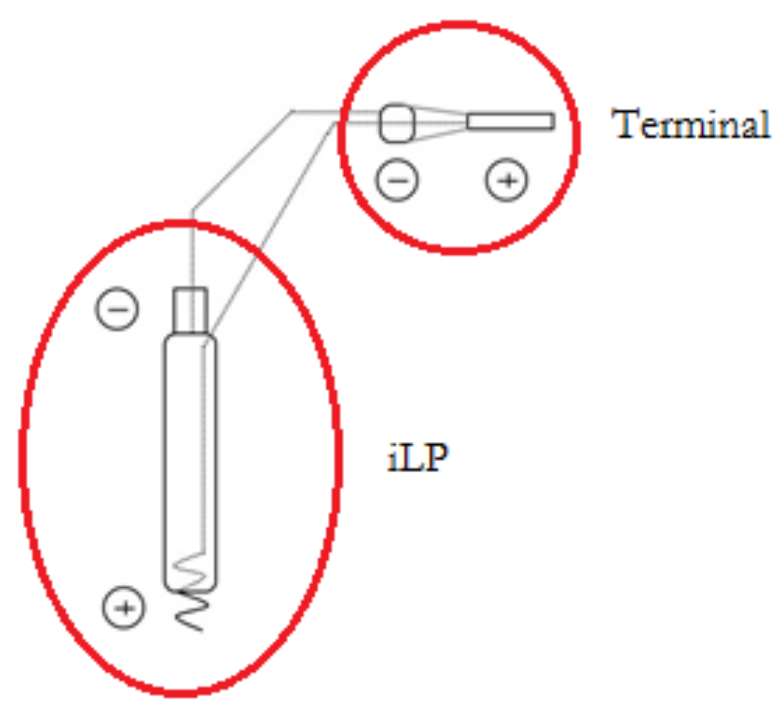

Figure 6: Diagram of iLP and connecting terminal

A photograph of the epoxy iLP prototype device is shown below in Figure 7. Both screw and hitch electrodes, as well as the white steroid-eluting collar around the screw, are visible. The steroid, Dexamethasone, is slowly released from the collar during chronic studies to suppress the foreign body immune response and to reduce scarring of the heart wall. The photograph also shows a titanium bar along the side of the device. Because titanium is radiopaque, the bars are clearly seen on $\mathrm{x}$-ray and allow the implanting surgeon to monitor how many turns are used to screw the device into place.

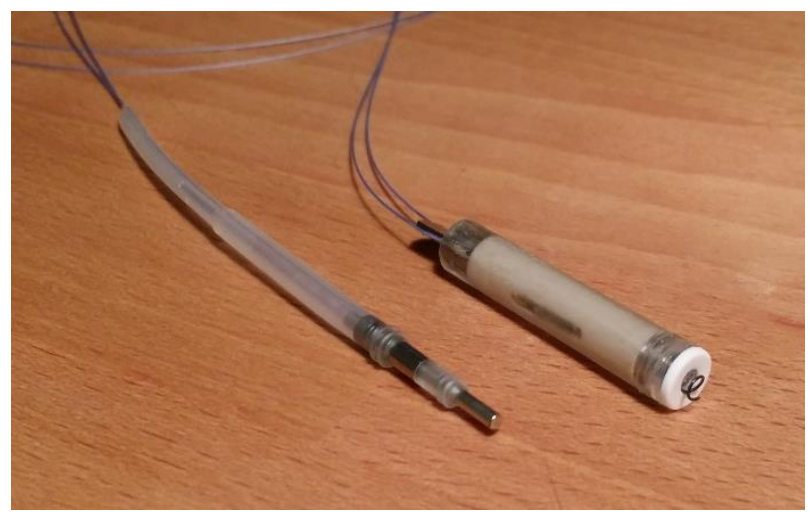

Figure 7: Epoxy iLP prototype device 


\subsection{Delivery Tool}

The prototype intracardiac device requires special tooling to navigate and fixate the device into the heart. Operated by a single surgeon, the delivery tool pinches onto the hitch of the device, steers the device into place, and applies torque to screw the device into the myocardium. Once the device is in place, the delivery tool disconnects from the wired prototype device and is extracted from the body without disrupting or applying tension to the DFT wires, leaving the iLP fixated securely near the apex of the right ventricle.

\subsection{Implantation Procedure}

The following describes the clinical procedure to implant an iLP into the right ventricle of an animal model. First, an incision is made at the site of the right external jugular vein in the neck. The vein is isolated and ligated distally from the heart. A guidewire is inserted into the jugular vein through a transvascular needle. Under fluoroscopic X-ray, the guidewire is navigated to the apex of the heart. Premature ventricular contractions (PVCs) seen on a surface ECG amplifier confirm that the guidewire is in the heart. Monitoring a surface ECG for PVCs is a practical means of determining whether a device is within the heart. Myocardial tissue is extremely sensitive to mechanical disturbance due to the sensitive nature of the leaky calcium ion channels. ${ }^{2}$

Next, a tissue dilator and sheath are passed over the guidewire, through the SVC, and into the heart. Fluoroscopy provides assurance that the tools are delivered to the heart and do

\footnotetext{
2 As an aside, during open-heart surgeries requiring cessation of cardiac contraction, the heart is completely arrested both pharmacologically and by packing the chest cavity with ice. When the heart is ready for reactivation, the ice is removed. Once the heart is warm, the surgeon needs only to flick the heart with a finger to activate the ion channels and jump-start the cardiac cycle.
} 
not penetrate the tissue. The dilator is designed to widen the vein prior to device delivery and reduce the likelihood of tearing the vessel as the tool passes through. The sheath is a stiff, thin-walled tube placed around the dilator. Once the dilator is in the heart, both the dilator and guide wire are removed through the sheath, leaving just the hollow sheath behind, providing an isolated and unimpeded pathway from outside of the body directly into the right ventricle.

The delivery tool and connected iLP are together passed through the sheath and into the right ventricle where, under fluoroscopy, the iLP is screwed into the apex of the heart. Once the iLP is fixated into the tissue, the delivery tool releases the device and is removed out through the sheath. Once removed, the sheath is removed from the body, leaving just the iLP behind. The jugular vein is tied off around the DFT wires to reduce blood effusion, leaving the IS-1 connector exposed to provide a direct electrical connection to the heart's interior.

\subsection{AC Signal Generation}

As in traditional impedance cardiography, monitoring changes in cardiac output from an intracardiac device requires a transthoracic signal to drive intracardiac impedance measurement at a given frequency. An isolated alternating current generator injects current through a pair of surface silver chloride ECG patches across the thorax. The patches are placed along the same vector as the implanted iLP device and separated by a distance twice the device's depth from the skin surface, marked with grey circles on the diagram below in Figure 8. 


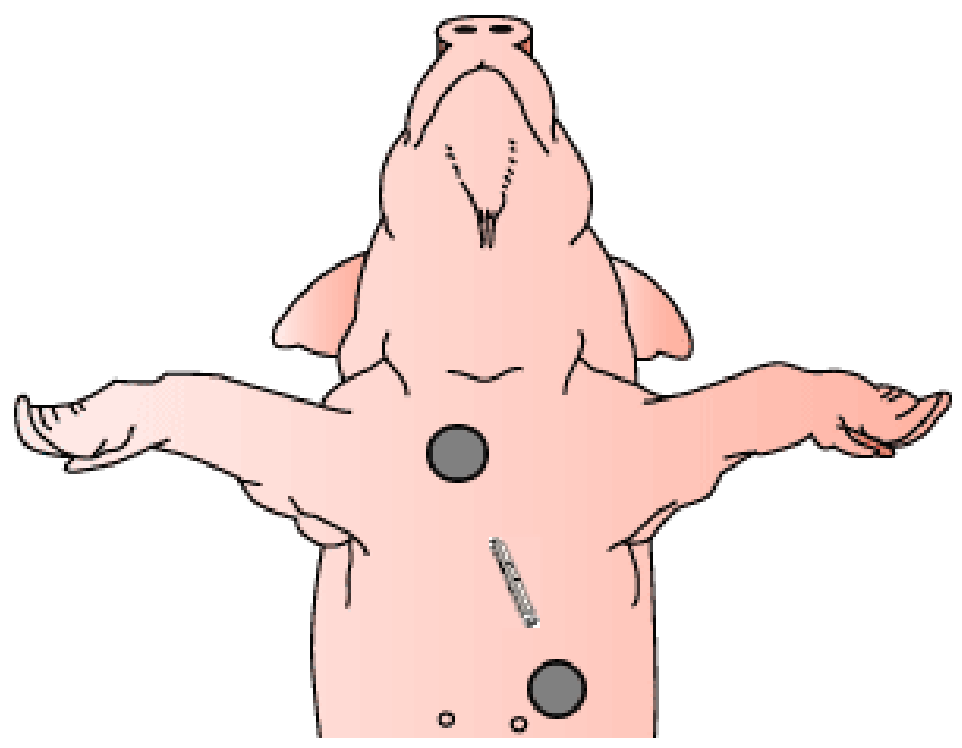

Figure 8: Approximate locations of signal injection electrodes indicated by grey circles. Grey circles indicate approximate current injection electrodes. iLP shown to scale

The objective of this thesis is to leverage the tools and resources presented in this chapter to acquire meaningful information regarding intracardiac dynamics. The following chapter describes an algorithm designed to monitor changes in intracardiac impedance to assess changes in cardiac stroke volume. 


\section{Algorithm Design}

Measuring changes in cardiac output requires an algorithm to derive and process the intracardiac impedance signal. To best design an algorithm for this task, a foundational understanding of the biophysical principles involved in this problem is desired. The following section describes the biophysical theory to explain how variation in intracardiac impedance is related to stroke volume.

\subsection{Theoretical Basis}

The iLP is implanted within the right ventricle. When the heart is relaxed, the device is surrounded by blood. As the heart contracts around the device, blood is ejected and the heart wall presses against the implanted iLP device, as evidenced by scar tissue formation on the heart wall following chronic implantation.

Blood and myocardium together provide current paths across the iLP device. As the heart contracts, the relative volumes of tissue and blood within the vicinity of the iLP change. As more-conductive blood is replaced by less-conductive myocardium during contraction, the impedance magnitude across the two iLP electrodes increases, increasing the sensed voltage amplitude across the iLP in accordance with Ohm's Law.

The characteristic electromechanical coupling between heart contraction and intracardiac impedance has been thoroughly established [24]. An example of electromechanical coupling derived from this investigation is shown in Figure 9. The intracardiac impedance increases after the QRS complex, indicating that the mechanical contraction expected after a QRS wave increases the impedance within the heart. 


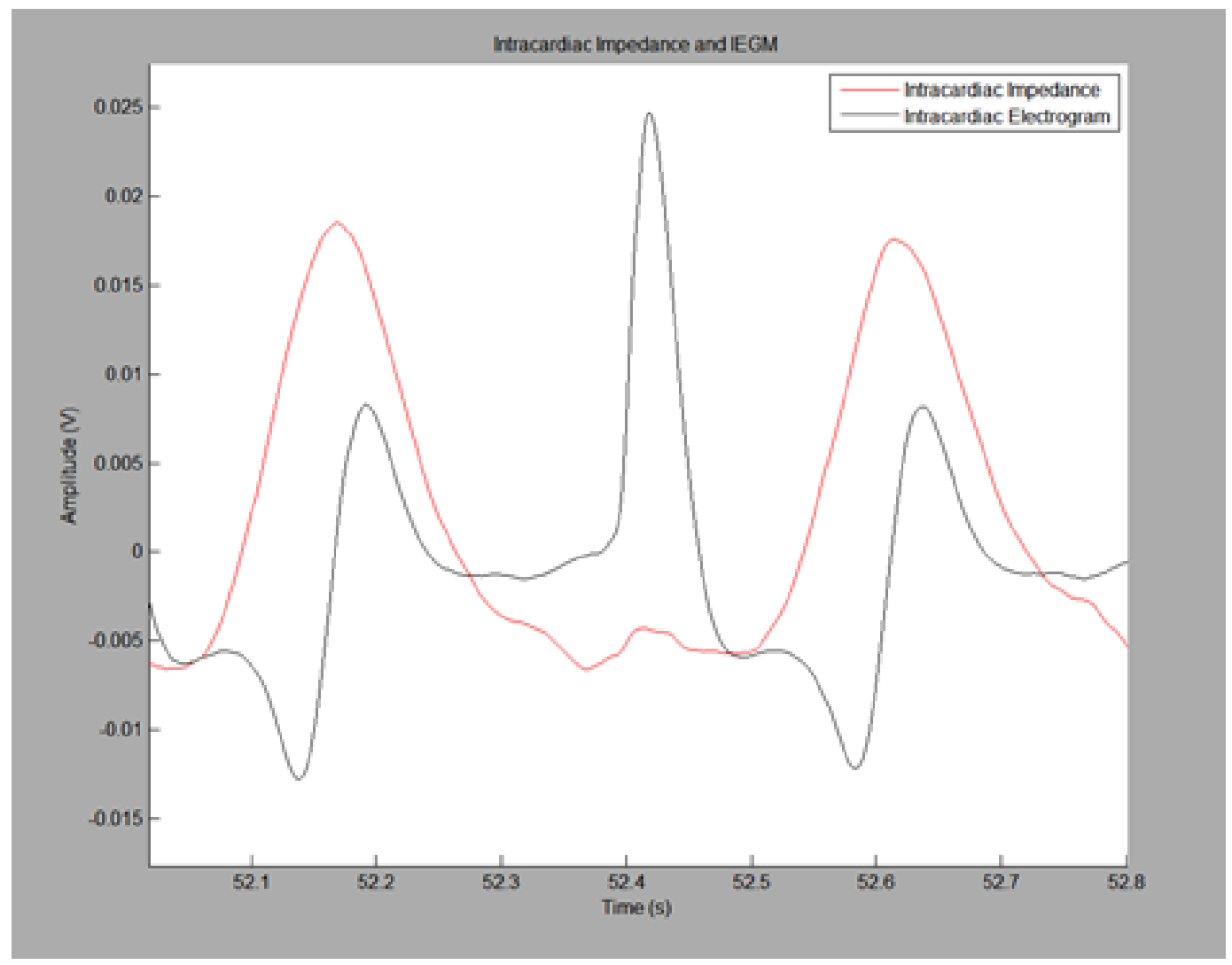

Figure 9: Single cardiac cycle visualized both with the intracardiac impedance curve and the IEGM

The time delay between the QRS wave and impedance increase is the electromechanical delay between electrical stimulation, the biochemical reaction cascade, and resultant mechanical contraction. As expected, the intracardiac impedance rises during systole and decreases during diastole. Both impedance and intracardiac electrogram (IEGM) are derived from the same sample of raw data collected from an implanted iLP.

The observation of sensed signal amplitude modulation discussed in the previous chapter provides evidence that an implanted iLP could theoretically extract information about intracardiac dynamics from the impedance signal driven by a high frequency voltage source, just as impedance cardiography monitors changes in thoracic impedance to estimate cardiac parameters. 


\subsection{Mathematical Model of Expected Impedance Changes}

To better predict the expected range of impedance change throughout the cardiac cycle across an implanted iLP, a mathematical model of time-varying cardiac impedance was developed. In this model, the heart is treated as a pair of nested cylinders. The outer cylinder represents the myocardium and the solid inner cylinder represents blood. Figure 10 shows the two cylinders surrounding a solid iLP model embedded within them. As the heart contracts, the volume of blood is reduced as blood is ejected out of the chamber. This physical behavior is modelled as a simultaneous contraction and thickening of the myocardium such that the cross-sectional area of the heart wall remains constant. The physical embodiment of the mathematical model is shown below in Figure 10.

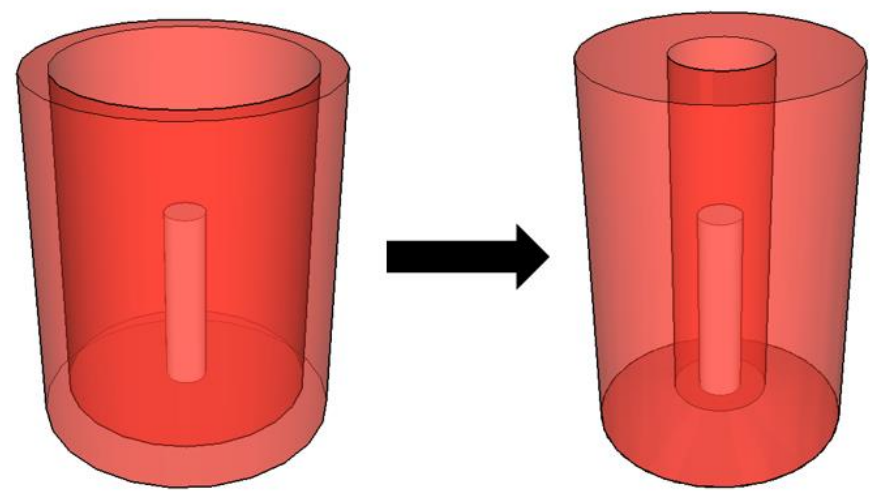

Figure 10: Simplified model of the right ventricle used to mathematically predict expected impedance changes

The specific geometric parameters of the physical model are derived from typical parameters associated with the human heart. The physical parameters used for this evaluation are tabulated below in Table 1. 
Table 1: Parameters utilized in the nested cylinder mathematical model

\begin{tabular}{lr}
\hline Diameter of iLP (mm): & 7.0 \\
Length of iLP (mm): & 35 \\
End Systolic Volume (mL): & 50 \\
End Diastolic Volume (mL) [25]: & 120 \\
Wall Thickness (mm) [26]: & 4.0 \\
RV length (mm) [27]: & 76 \\
Blood Resistivity $(\boldsymbol{\Omega} / \mathbf{m}):$ & 1.36 \\
Myocardium Resistivity $(\boldsymbol{\Omega} / \mathbf{c m}):$ & 206 \\
\hline
\end{tabular}

The physical geometry of the mathematical model is coupled with known electrical properties of human tissue to produce a simplified model of the right ventricle.

\subsubsection{Tissue Resistance and Reactance}

Human biological tissue is complex, consisting both of extracellular components, cells, and intracellular components. Modeling biological tissue is slightly more complex than typical electrical components as cell walls have been shown to act as biological capacitors, separating charged ions between in the inside and outside of an individual cell. Biological tissue is typically electrically modelled with both pure resistance elements and complex capacitance elements which accounts for both the purely resistive interstitial fluid between cells as well as the chargeseparating capacity of cellular walls [28]. Mathematically tissue modeling must consider both real and imaginary components of complex impedance.

Electrical characteristics of myocardial tissue have been extensively investigated. Characterizing intracardiac dynamics requires understanding the complex nature of intracardiac impedance. Considering capacitance, the impedance of complex biological material includes an imaginary component and an associated phase angle to account for both the real resistance and imaginary reactance of tissue. According to recent studies, healthy myocardial tissue is characterized as having an impedance magnitude of $206 \pm 40 \Omega \cdot \mathrm{cm}$ and a phase angle of $-8.8 \pm 0.7^{\circ}$ at $100 \mathrm{kHz}$, the frequency of AC signal injection [29]. Data 
characterizing healthy myocardial tissue impedance at the frequency of carrier injection throughout the cardiac cycle was not found. However, because myocardial impedance is dominated by the real component of impedance, the mathematical model presented here assumes that the capacitance remains constant throughout the cardiac cycle and that impedance magnitude is dependent on the geometry of the myocardium.

The resistance of a three dimensional material can be derived from Pouillet's Law by relating the known electrical resistivity of a material and its geometry. Pouillet's Law is shown in the equation below where $R$ is the net electrical resistance, $\rho$ is the material's known electrical resistivity, $l$ is the length of the material, and $A$ is the material's cross-sectional area.

$$
R=\rho \frac{l}{A}
$$

This model assumes that both the myocardium and the blood can be modeled as nested cylinders whose height and electrical resistivity remain constant while the crosssectional area changes throughout the cardiac cycle. Resistance is calculated between the two electrodes of an embedded iLP. This model only considers changes in real impedance of the heart. By treating the impedance magnitudes of blood and myocardium as parallel resistors, an equivalent resistance is determined. The impedance magnitude of both tissues and their equivalent resistance as a function of stroke volume are shown below in Figure 11. 
Intracardiac Impedance Model Prediction

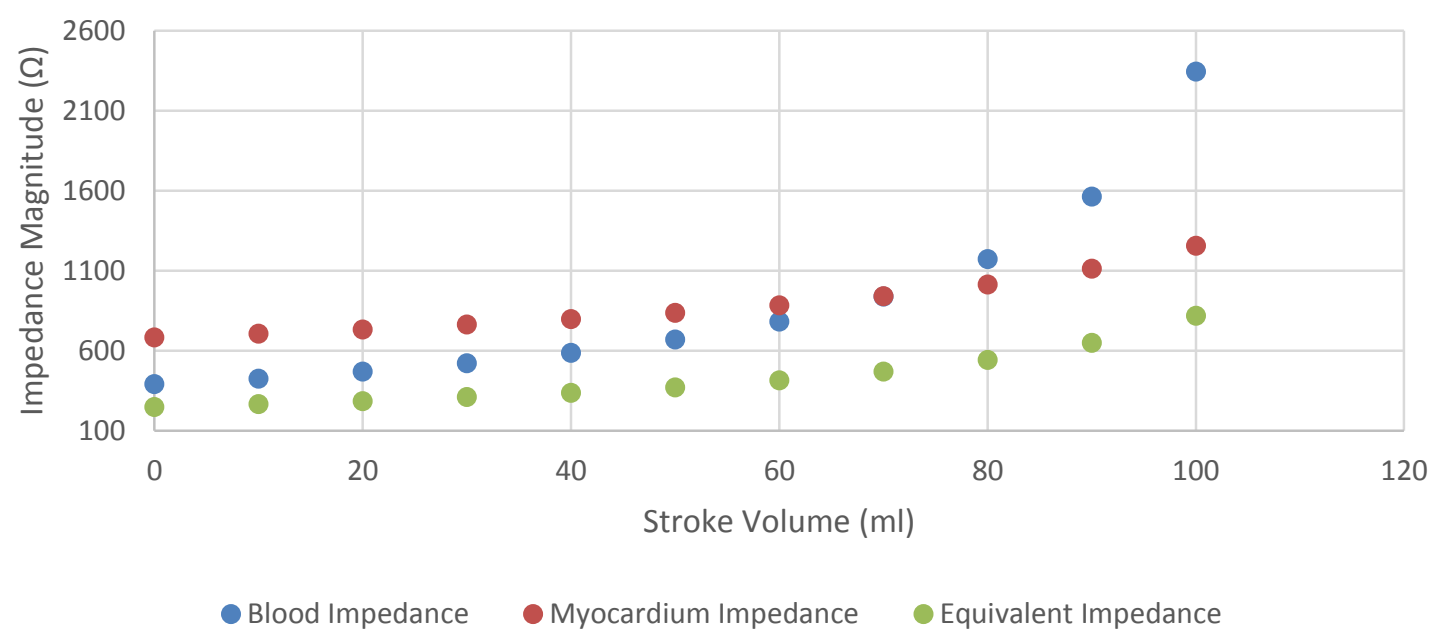

Figure 11: Mathematical prediction of resistances for blood, myocardium, and their functional equivalent

The above figure illustrates how the functional resistance across the iLP is expected to change over the course of the cardiac cycle. From the above data, a nominal stroke volume of $70 \mathrm{ml}$ would correspond to an increase in device resistance of approximately $221 \mathrm{ohms}$ or a $189 \%$ increase in resistance from end diastolic volume to end systolic volume.

A simplified schematic of the body conductor model is shown below in Figure 12. As shown in the following schematic, an offset resistance associated with the path from the current injection to the iLP is expected. Additionally, alternative current paths exist away from the iLP. In a body, these could be any of the fluid-filled, more conductive great vessels such as the aorta, the superior vena cava, and the inferior vena cava capable of conducting current away from the heart. How significantly alternative current pathways affect the overall equivalent resistance of the body is difficult to predict and likely varies significantly between individuals. For the purpose of simplification, these variables are excluded from this model. 


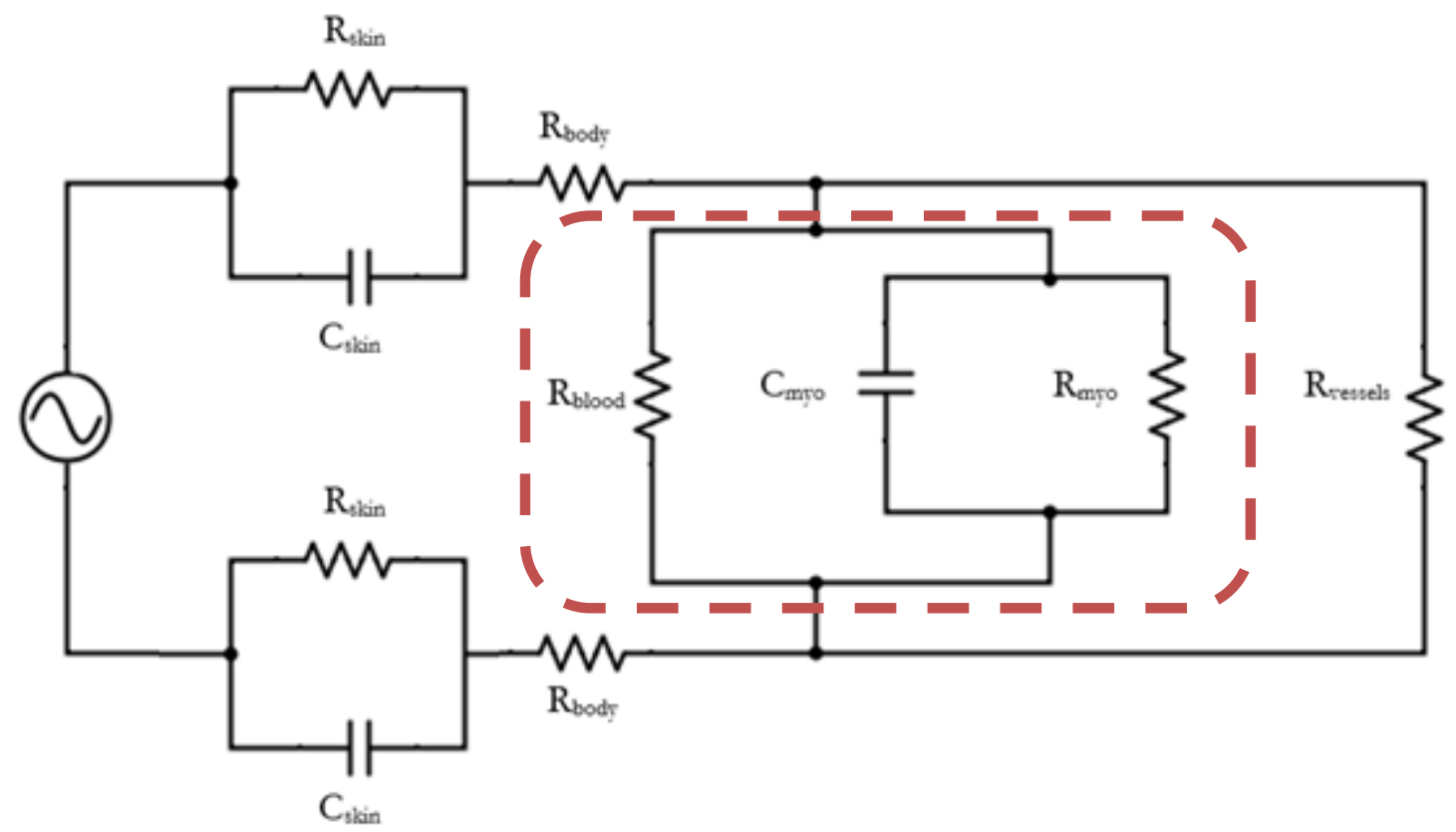

Figure 12: Model circuit diagram of the body where elements specific to the heart are circled in red

As shown in the above diagram, the variable resistance of the two conducting mediums within the heart has a direct impact on the measured voltage across the iLP. However, the presence of alternative current pathways and the varying degrees that they shunt electrical current away from the iLP throughout the cardiac cycle are difficult, if not impossible, to predict or model accurately. Because these alternative current pathways are not accounted for in the nested cylinder model, it is reasonable to presume that the model overestimates the expected impedance change as the presence of alternative current pathways would lower the equivalent resistance of the whole body conductor and reduced sensed voltage amplitude at the iLP.

\subsection{The Z-Cardio Algorithm}

The algorithm designed to process the time-varying intracardiac impedance profile and provide a measurement of cardiac output was designed in MATLAB and is hereby referred to 
Z-Cardio. The Z-Cardio algorithm is designed to monitor changes in systolic intracardiac impedance through the process detailed in the high-level flow diagram below in Figure 13.

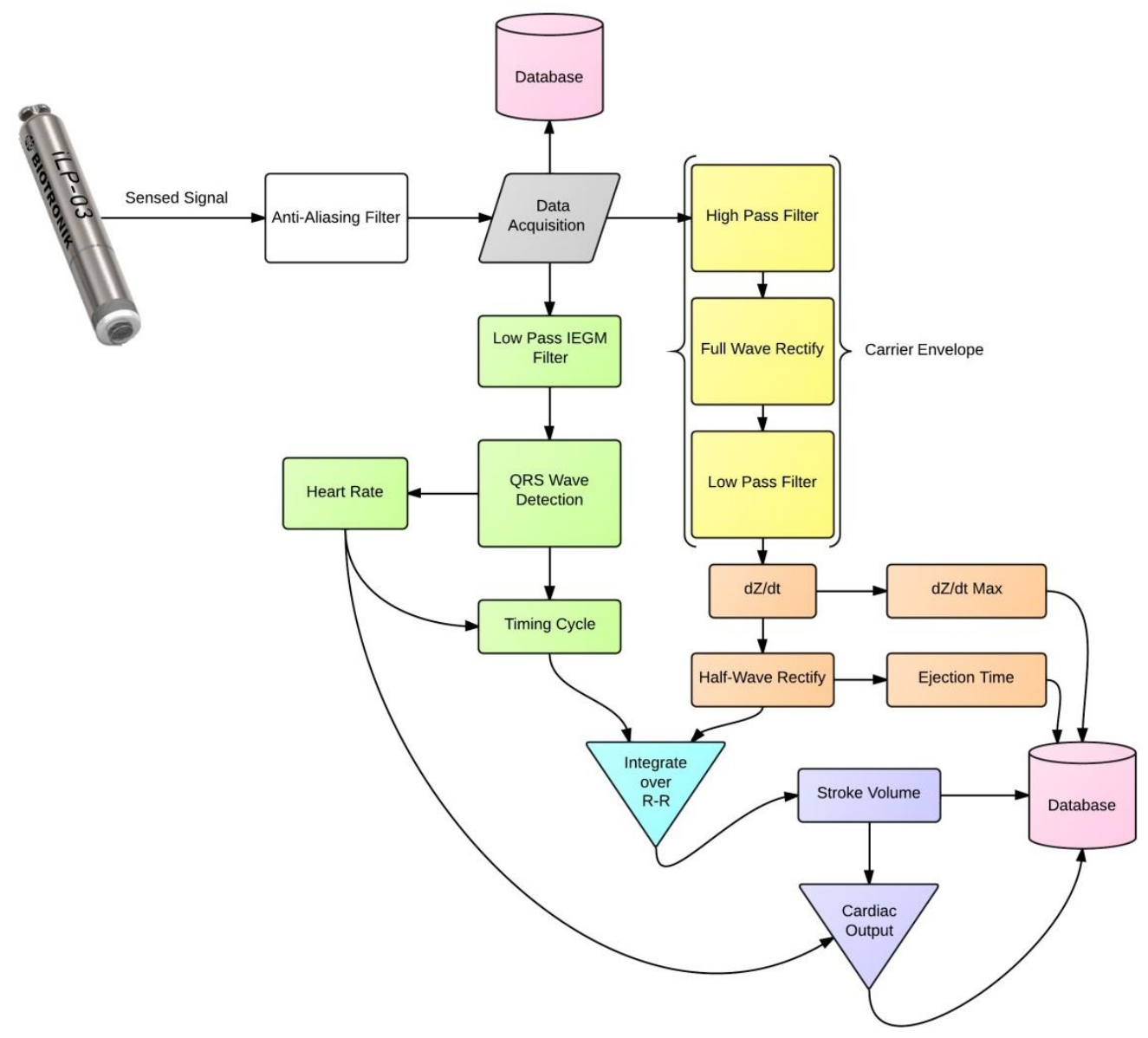

Figure 13: High-level flow diagram of the Z-Cardio algorithm

The purpose of the algorithm is to process the measured voltage at an iLP to render meaningful clinical data pertaining to intracardiac dynamics. First, a device is implanted in the right ventricle. A pair of surface electrodes generates a high frequency voltage source across the thorax to drive the intracardiac impedance signal at a known frequency. Raw voltage data is collected from the two electrodes of the iLP from within the heart.

Collected data is processed in two parallel paths. The first stage, shown in green above, 
isolates the IEGM from the raw voltage signal by applying a low-pass filter to remove the high-frequency voltage source and other irrelevant physiological noise. The QRS is detected by a simple threshold-crossing criterion where a QRS marker is designated at the point where the IEGM first crosses a positive threshold value. The rate that the QRS is detected is the heart rate; the interval between two QRS events is the R-R interval, or the heart rate period. While heart rate could be derived from the intracardiac impedance curve, the heart's electrical activity may be a more reliable means of estimating heart rate periodicity because it relies on less complex signal processing.

The raw data is also processed to extract the envelope of the generated voltage source undergoing amplitude modulated throughout the cardiac cycle. As described previously, the cardiac cycle modulates the impedance across the iLP by alternating the relative ratio of blood and myocardium, each possessing unique conductivities. As the contracting heart changes impedance across the device, the measured voltage will change according to Ohm's Law.

The envelope detection process is colored yellow in the flow diagram above. First, a digital high pass filter removes any physiological electrical activity, isolating the voltage source and removing any $\mathrm{DC}$ offset from the raw voltage signal. The signal is full wave rectified and filtered with a low pass filter to produce a clean envelope signal, which represents the intracardiac impedance signal shown in yellow in the top plot of Figure 14 below. 


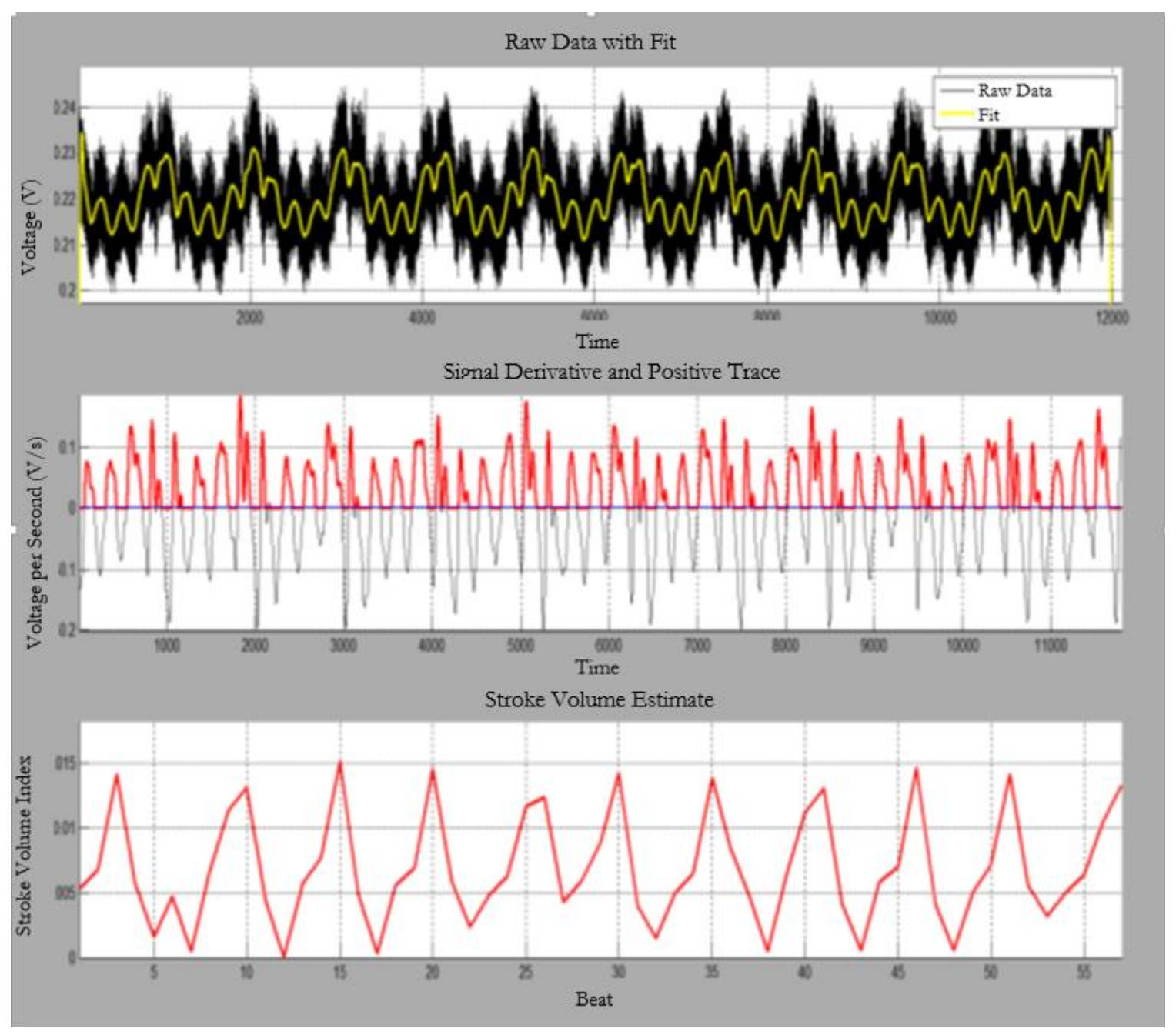

Figure 14: Illustration of the Z-Cardio algorithm processing cascade. The top plot shows the raw data in black and the low-pass trend in yellow. The middle plot shows the differential of the yellow trace from the top plot, and highlights the positive portion (half-wave rectification) of the curve in red. The bottom plot shows the integral of the red curve shown in the middle plot for each beat.

The voltage source envelope signal is differentiated with respect to time, referred to here as $\mathrm{dZ} / \mathrm{dt}$. The first time derivative describes how the rate of change in intracardiac impedance varies with time. Of specific interest is how the intracardiac impedance signal changes during systole, when the heart is contracting. Only during systole is blood ejected from the heart. Because the rate of intracardiac impedance change is positive during systole, only the positive portion of the $\mathrm{dZ} / \mathrm{dt}$ curve is of interest, highlighted in the middle plot of 
Figure 14. The time duration of each positive $\mathrm{dZ} / \mathrm{dt}$ segment is the time when impedance is increasing and blood is being ejected, or the ventricular ejection time (VET).

To estimate stroke volume, the area under each of the positive $\mathrm{dZ} / \mathrm{dt}$ segments is integrated over the R-R interval for each beat. This stroke volume index is shown in the bottom plot of Figure 14. Notice how rhythmic fluctuations in the stroke volume index correspond to the respiration rate, known to have a significant impact on cardiac stroke volume [30]. As the diaphragm contracts downwards to inspire, thoracic pressure is reduced, increasing ventricular preload and increasing stroke volume.

By the two processes described above, both heart rate and a stroke volume index can be estimated. Their product is a cardiac output index. This value is assumed to be correlated to a measured cardiac output, requiring the employment of a gold standard calibration metric for true cardiac output estimation.

The injected signal is an alternating voltage signal at $100 \mathrm{kHz}$. The amplitude was adjusted such that the amplitude of the sensed signal at the implanted device was approximately $15 \mathrm{mV}$. The amplitude of the injected signal is adjusted to ensure that the amplitude of the sensed signal does not rail the input to the DAQ. A passive RC anti-aliasing filter was used at the input of the DAQ to attenuate frequencies above the Nyquist frequency. Because data was sampled continuously at $400 \mathrm{kHz}$, the Nyquist frequency is $200 \mathrm{kHz}$. To ensure that frequency spectra at and above the Nyquist frequency are sufficiently attenuated, the RC anti-aliasing low-pass filter was designed with a corner frequency at $160 \mathrm{kHz}$. A second high-pass RC filter was cascaded with the RC anti-aliasing low-pass filter to remove any DC offset of the signal detected by the implant. The corner frequency of the high-pass front-end filter is $1 \mathrm{~Hz}$, sufficient to attenuate the DC component of the input signal yet low enough to 
not substantially distort the desired electrical activity of the heart.

In this design, the low-pass and high-pass filters are designed in MATLAB as second order Butterworth filters. Second order Butterworth filters are desirable because they exhibit zero ripple in the passband frequency range, have relatively steep roll-off of $40 \mathrm{~dB} / \mathrm{decade}$, and are easily implemented in analog circuitry. While analog filter realization is not a direct design requirement of this thesis, any effort to implement Z-Cardio into implantable hardware would benefit from translating the digital filters into analog filters on a printed circuit board to offload as much computation and battery power demand from the implant device as possible.

The QRS wave detection block places markers over instances of a QRS wave in the IEGM. A simple amplitude threshold criterion determines the presence of a QRS wave. The programmed amplitude is dependent on the amplitude of the detected signal and is selected by the user to optimize detection consistency.

Validation of the Z-Cardio algorithm requires a living model and in vivo experimentation protocol. To support justification for animal experimentation, illustrating that the Z-Cardio algorithm is capable of monitoring changes in stroke volume in a benchtop simulator is desired. A simulator designed to model expected impedance changes within an animal model used to assess the Z-Cardio algorithm was constructed. 


\section{Simulator Design Requirements and Verification}

To conform to both national and international regulations governing the medical device industry, validation of the Z-Cardio algorithm requires the use of large animal models in well-controlled, well-documented, and well-supported clinical studies. The use of animals in medical device testing, while necessary for ensuring the safety and well-being of the patient, should be minimized to reduce both financial costs and costs associated with animal sacrifice. While a simulator cannot completely replace the need for live animal study, a simulator can provide a valuable tool to validate the Z-Cardio algorithm concept and to validate the technology as much as possible prior to animal study. D.I.C.E., the Dynamic Intra-Cardiac Emulator, was designed and constructed to support early stage design validation.

\subsection{Design Requirements}

To provide a useful tool to validate the Z-Cardio algorithm, an intracardiac impedance simulator must fulfill each of the following design requirements.

\subsubsection{Low-Cost and Rapid-Development Design}

Establishing bench-top proof-of-concept as soon as possible more easily facilitates clinical study protocol planning. Constrained by a limited development budget, the simulator must be low-cost and should be designed and constructed in a reasonably short time frame. A simple and elegant design solution is desired.

\subsubsection{Controllable and Measurable Simulator Stroke Volume}

The purpose of the simulator is to facilitate development of an algorithm capable of monitoring changes in stroke volume. Therefore, any estimation made by the algorithm must be correlated to the true stroke volume of the simulator. To accommodate a range of stroke 
volumes and to provide a standard for the Z-Cardio algorithm, the simulator's stroke volume must be both controllable and measurable.

\subsubsection{Voltage Source Generation Across the iLP}

Measuring intracardiac impedance requires a driving source. In an animal or human subject, a high frequency voltage source is generated across surface patch electrodes. The simulator must accommodate a similar means of voltage generation such that the voltage is detectable by an iLP.

\subsubsection{Blood and Myocardial Tissue Emulation}

When the heart contracts, the composition of the tissue surrounding the iLP changes as the chamber ejects blood and contracts myocardial tissue. Because the iLP is located entirely within the heart, assuming that the local impedance profile is modulated primarily by the alternating ratio of both blood and myocardium within that chamber is reasonable.

When the heart is in peak diastole, a large volume of conductive blood provides highconductivity pathways between the two iLP electrodes. By reducing the impedance magnitude across the device during diastole, the amplitude of an observed voltage driven by the voltage source would be lower, in accordance with Ohm's Law. As the heart begins to contract, the muscular, less conductive heart wall approaches the device, effectively replacing the moreconductive blood with less-conductive myocardium within the heart. At peak systole, when the heart is least filled with blood, the myocardium most completely surrounds the iLP, maximally impeding current flow between the iLP electrodes and thereby maximizing the sensed voltage across the iLP. The D.I.C.E. simulator must therefore model the conductivities of both blood and myocardium and control how the relative ratio of each of the tissues changes around an iLP during contraction. 


\subsubsection{Realistic Impedance Modulation}

The purpose of the simulator is to recreate an impedance profile similar to what would be expected from within a living subject's heart. While an exact replication of intracardiac impedance is desired, the simulator must at the very least increase and decrease impedance magnitude during systole and diastole, respectively, to be considered an acceptable simulation. As previously discussed during mathematical model description, complex tissue impedance is composed of both real resistance and imaginary reactance components. However, studies suggest that the impedance magnitude of myocardial tissue is dominated by the purely resistive component of impedance. Because the Z-Cardio algorithm is designed to monitor changes in amplitude and not changes in signal phase, only impedance magnitude is calibrated for the purpose of this simulation.

\subsection{D.I.C.E. Design}

D.I.C.E. was designed to simulate the natural rhythmicity of intracardiac impedance expected within a living subject's heart during cardiac contraction. The design of the simulator is described by two major design components. The emulated tissue design describes how appropriate tissue analogs were created to simulate the electrical conductivity of blood and myocardial tissue. The mechanical design describes the physical simulator, how it incorporates the emulated tissue, and the means to control simulated stroke volume.

\subsubsection{Emulated Tissue Design}

The D.I.C.E. bench-top simulator was designed to modulate impedance across the iLP by controlling the relative ratio of blood and myocardium surrounding the iLP device during contraction. Because both blood and myocardium are organic tissues, non-living 
alternatives were developed to eliminate the need for tissue acquisition and preservation. To ensure that alternative analogs for blood and myocardium were appropriate for the design purpose, each alternative was calibrated to known electrical properties of their respective natural tissue. However, artificial analogs lack the cell walls present in biological tissue that introduce capacitive reactance. To simplify the design of emulated tissue, artificial tissue impedance magnitude is calibrated independent of resistance and capacitive effects. We assume impedance of the tissue analog is dominated by pure resistance.

Saline solution is marketed as a tissue analog. While saline solution is available for purchase, creating original saline solutions provides greater control over electrical conductivity, essential when calibrating saline to known human tissue properties. Phosphatebuffered saline (PBS) tablets were chosen because they are cheap, readily available, and provide greater control over saline concentration and electrical conductivity than off-the-shelf saline solutions. Mixed with distilled water, PBS tablets dissolve to produce an isotonic salt saline solution whose osmotic concentration, ion concentration, and $\mathrm{pH}$ match those of human tissue. Controlling the concentration of PBS controls the ionic concentration and electrical conductivity of each solution.

Both blood and myocardial solutions were produced by measuring solution impedance magnitude at a known frequency while mixing PBS with distilled water until target values were reached. Figure 15 plots the target conductivities of blood and myocardium saline solutions at the nominal frequency band of voltage generation [31]. 


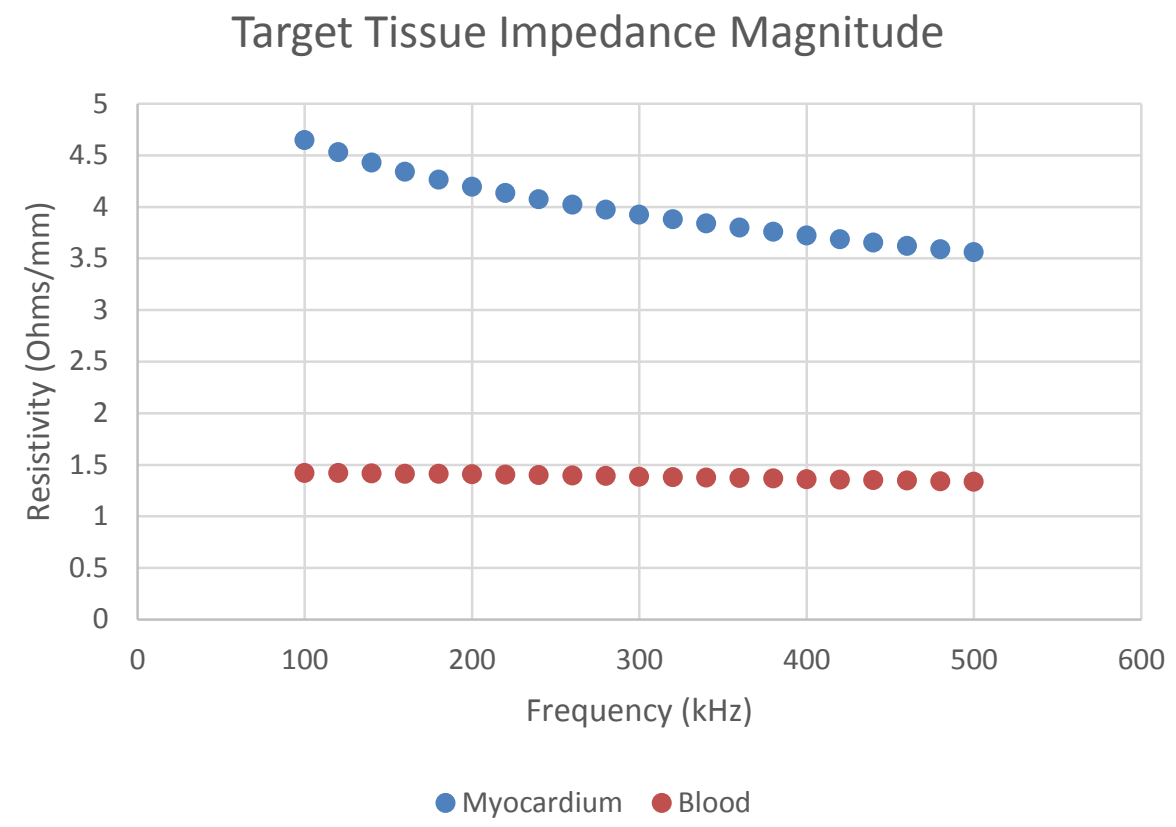

Figure 15: Resistivity of blood and myocardium over the nominal voltage generation frequency band

Both blood and myocardial analogs were incorporated into a mechanical device that prevented the two solutions from mixing while mimicking the contraction dynamics of a natural heart.

\subsubsection{Mechanical Design}

A mechanical design requires both the artificial blood and myocardium calibrated saline solutions be separated to prevent mixing and compromising their unique conductivities. The interface chosen between the two solutions is a silicone bladder with a wall thickness of $5 \mathrm{~mm}$ and an internal volume of $100 \mathrm{~mL}$, approximately the volume of a healthy right ventricle [32]. Silicone is a good candidate for a solution interface because it is low-cost, non-porous, flexible, and retains its shape after plastic deformation.

The top outflow tract of the silicone bladder was connected to a graduated cylinder. The cylinder is incorporated to provide the user a convenient means to measure the volume 
of fluid ejected from the silicone bladder. The difference in meniscus height within the cylinder during contraction is the system stroke volume.

The blood-filled silicone bladder was submerged into a container of myocardium saline. The container was built from Plexiglas, a light-weight, transparent, and inexpensive plastic that is easily made water-tight. The draft design drawing of the Plexiglas housing is found in Appendix A: D.I.C.E. Design Drawing. The housing was designed with size and weight in mind, thereby permitting portability as necessary.

The simulator must consistently compress the silicone bladder in a manner similar to how the right ventricle deforms during cardiac contraction. A uniform deformation within the simulator requires a uniform positive pressure increase of the myocardium saline. An increase in pressure collapses the silicone bulb and ejects blood saline up the graduated cylinder. To induce a positive pressure increase in the myocardium saline, a compressible hand-pump was connected to the side of the Plexiglas housing and filled with myocardial saline. The hand pump has an internal volume of $150 \mathrm{~mL}$, slightly greater than the volume of a healthy right ventricle, necessary to replicate a stroke volume range between 55 and $100 \mathrm{ml}$, or the nominal stroke volume range expected within a healthy human heart [33]. When the hand-pump is compressed, the resulting pressure deforms the silicone bladder and ejects blood saline up the graduated cylinder, ensuring that the simulator stroke volume is both controllable and measureable.

In a typical clinical setting, the high frequency voltage potential would be generated across a pair of surface patch electrodes located on the subject's skin. Because Plexiglas is a poor conductor, a voltage potential generated from outside of the Plexiglas would experience its greatest drop across the Plexiglas wall, making the signal more difficult to detect at an iLP. 
Instead of generating the voltage from outside the Plexiglas, two wire electrodes were suspended in the myocardium solution through which the high frequency voltage was generated, thereby ensuring that the high frequency signal is more easily detected by the iLP.

\subsection{D.I.C.E. Prototype}

Following the proposed design plan described above, a functional prototype of the D.I.C.E. simulator was constructed. A photograph of the experimental simulator and its associated hardware, including a function generator and a laptop running MATLAB, is shown below in Figure 16.

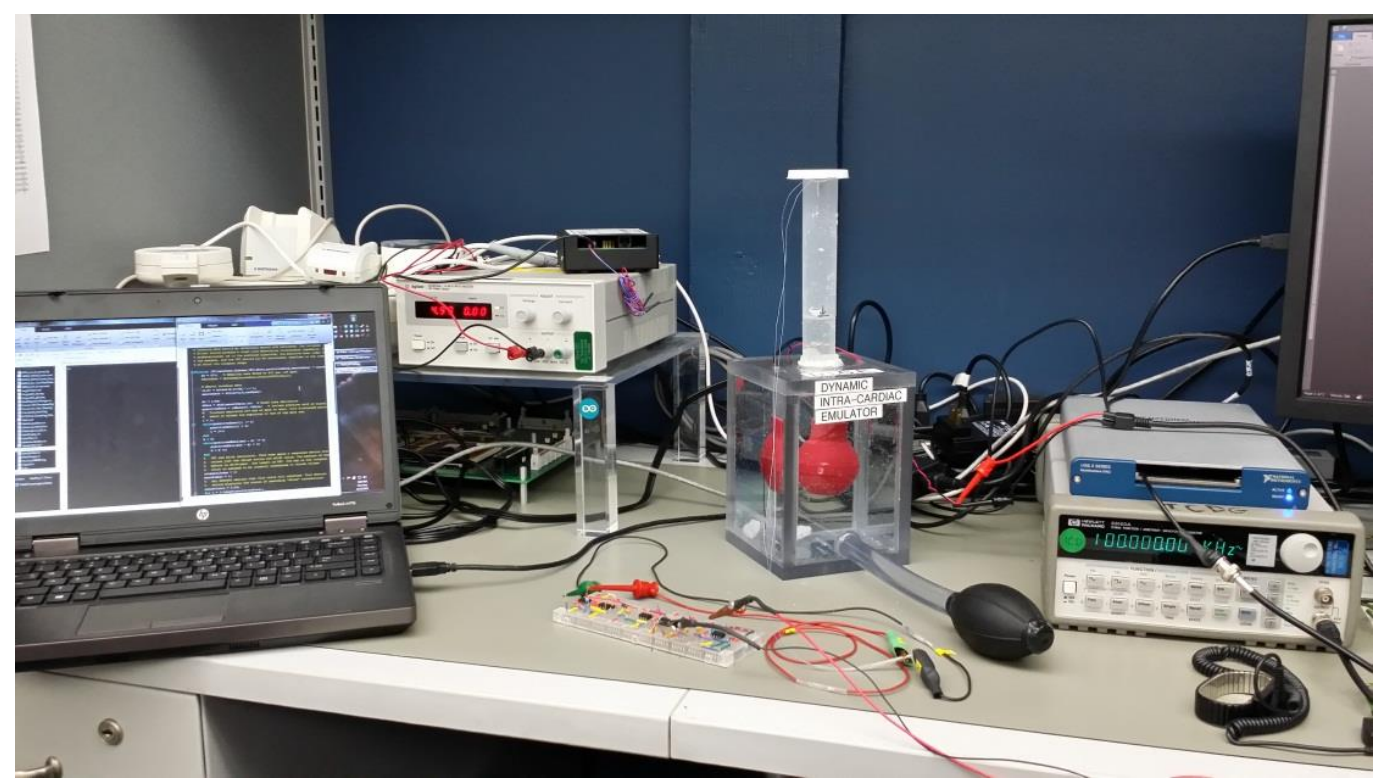

Figure 16: D.I.C.E. and associated hardware

Two close-up photographs of the D.I.C.E. simulator are shown below in Figure 17.

Note the red silicone bladder filled with blood saline, the graduated cylinder from where stroke volume is measured, and the wires hanging out the top of the graduated cylinder that connect to an iLP embedded within the silicone bladder. The red and black wires through the top of the Plexiglas housing are used for voltage generation. The compressible hand-pump is 
connected to the simulator by the clear tubing shown near the bottom of each photograph. The black compressible hand-pump is seen more clearly in the preceding photograph above in Figure 16.
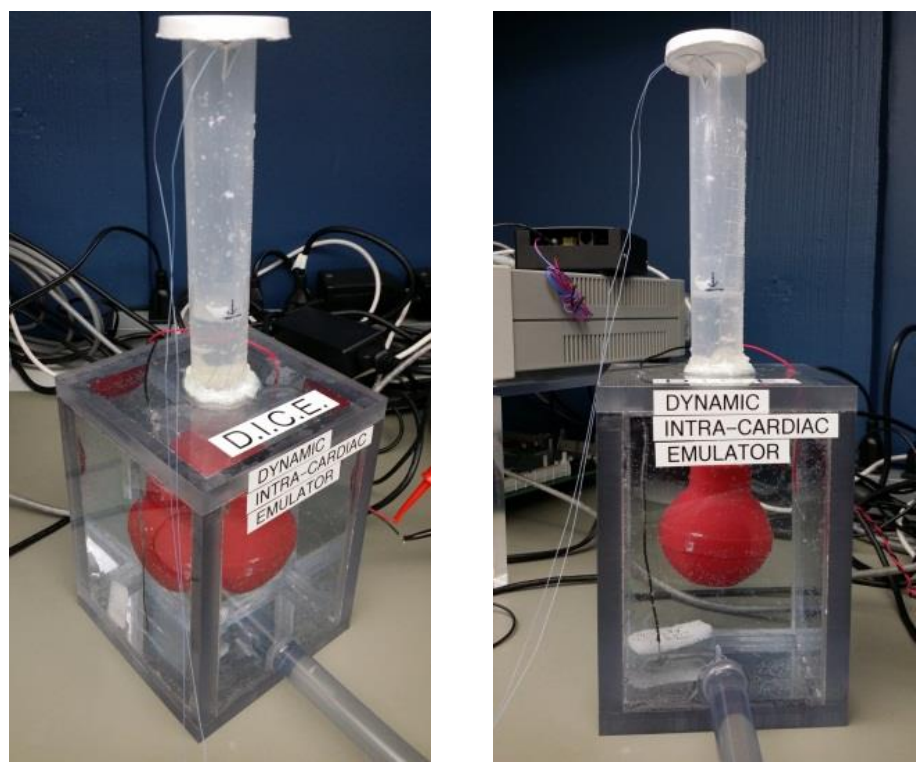

Figure 17: Close-up photographs of the D.I.C.E. simulator

The contraction dynamics of the simulator are illustrated in the series of three photographs shown below in Figure 18. The photographs depict the progressive deformation of the silicone bulb during the course of contraction within the simulator. The meniscus within the graduated cylinder rises as the silicone bulb deforms, indicated below with black arrows. 


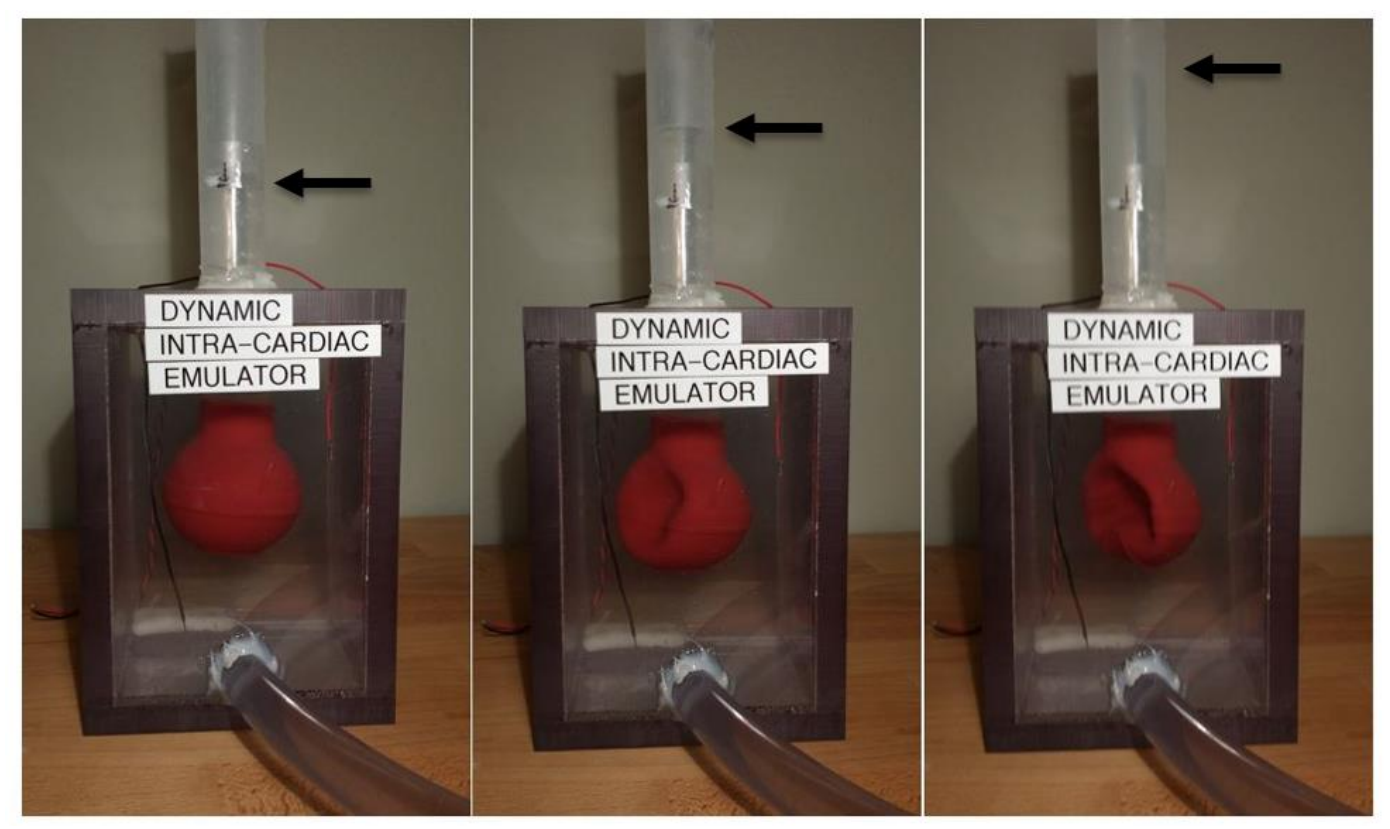

Figure 18: Simulator contraction dynamics of increasing stroke volumes indicated in the graduated cylinder by black arrows

\subsection{Design Verification}

The prototype D.I.C.E. simulator was designed to fulfill the requisite design requirements. The design was planned and executed in a reasonable time frame and within a limited budget, thereby fulfilling the first design requirement. The design allows for a controllable and measurable stroke volume through the use of a hand-pump and a graduated cylinder, thereby fulfilling the second design requirement. A high frequency voltage source can be generated across a pair of electrodes submerged within the simulator such that the signal is detectable by an iLP, thereby fulfilling the third design requirement. Two uniquely calibrated saline solutions designed to match known electrical conductivity constants of human tissues were developed and incorporated into the D.I.C.E. design, thereby fulfilling the fourth design requirement.

To verify that the D.I.C.E. simulator fulfills the fifth design requirement, the following 
assessment sought to quantify impedance change across an iLP within the simulator as a function of the simulator stroke volume. The D.I.C.E. system's stroke volume was measured by observing changes in meniscus height within the graduated cylinder. The impedance across the iLP was measured by an impedance meter calibrated to the frequency of voltage generation. The linear model associating simulator stroke volume and impedance across the iLP is shown below in Figure 19.

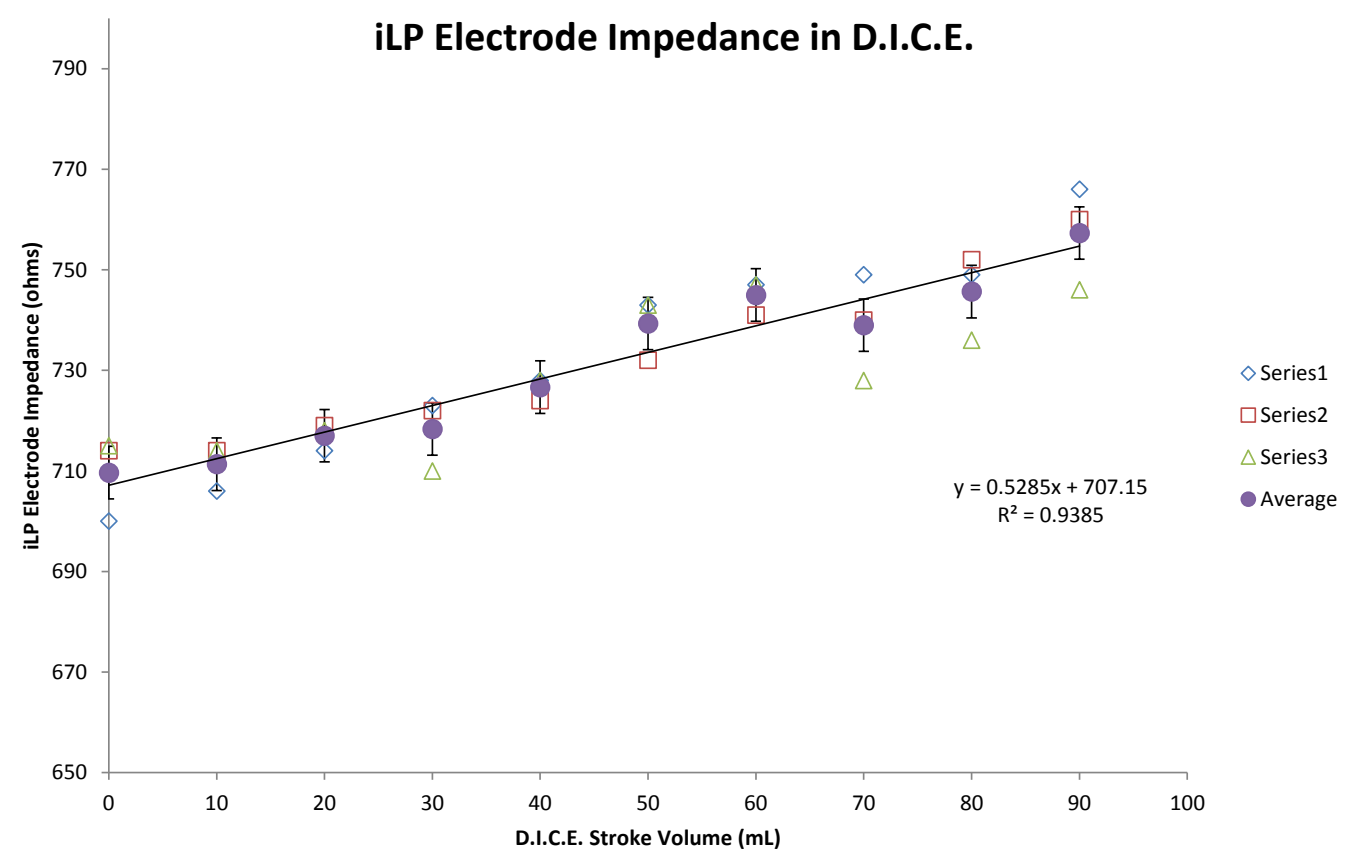

Figure 19: Regression analysis between D.I.C.E. stroke volume and impedance across the iLP

The above figure illustrates a linear relationship between D.I.C.E. stroke volume and observed changes in impedance across an embedded iLP device. The linear relationship between impedance (ohms) and stroke volume (mL) is $0.5285 \mathrm{ohms}$ per milliliter. These results contrasts with the mathematical expectation as predicted by the nested cylinder model. The expected change in impedance associated with a $70 \mathrm{ml}$ stroke volume according to the mathematical model was $220 \Omega$ whereas in the D.I.C.E. simulator the change was 
approximately $22 \Omega$. This significant deviation could result from poorly mathematically modeling alternative current pathways throughout the body during the cardiac cycle or from other physical behavior not accounted for by the mathematical model that nevertheless manifest within the D.I.C.E. simulator. Despite less significant changes in impedance across the iLP device than expected, the impedance profile nevertheless reflects reasonable expectations of a live animal model, thereby fulfilling the fifth and final design requirement. 


\section{Simulator Analysis, Results, and Discussion}

Following the assembly of the prototype D.I.C.E. simulator, performance of the ZCardio algorithm was evaluated by comparing the measured simulator stroke volume to the Z-Cardio stroke volume estimate that is derived from the impedance profile of the simulator. In this analysis, the Z-Cardio algorithm needs only measure changes in stroke volume instead of cardiac output as monitoring heart rate from within a living heart is a trivial challenge.

\subsection{Algorithm Performance and Stroke Volume Comparison}

The following assessment of the D.I.C.E. simulator sought to evaluate how the stroke volume estimation made by the Z-Cardio algorithm compared to the stroke volume measured from the simulator. The following study sampled data continuously from the embedded iLP device while the D.I.C.E. simulator contracted across a range of physiologically appropriate stroke volumes. Figure 20 illustrates the impedance profile during five D.I.C.E. contractions at five stroke volumes, stepping up in stroke volume amplitude every five contractions. The first component of the algorithm that isolates the intracardiac impedance signal from the high frequency voltage signal envelope is applied to the raw data. 


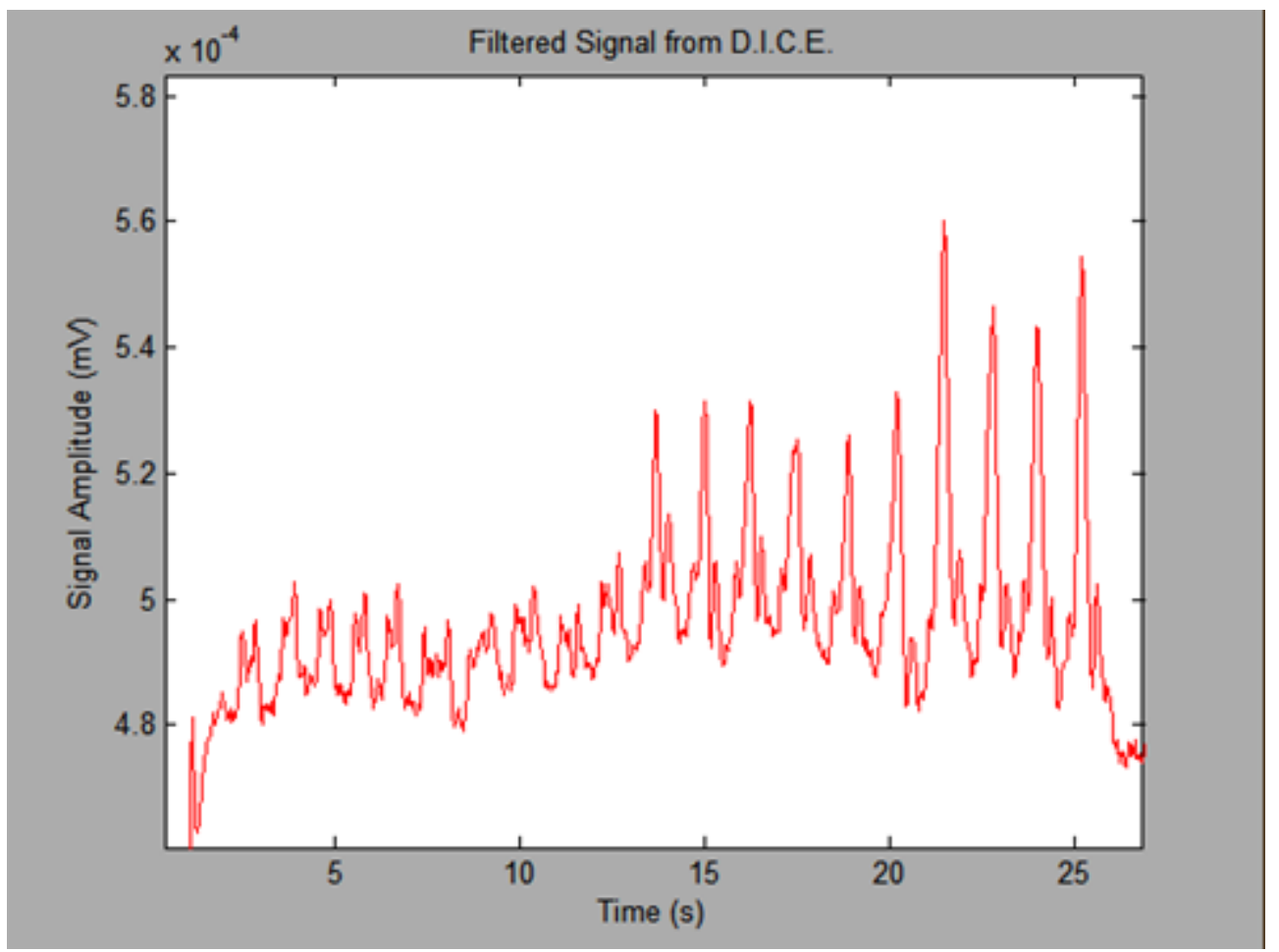

Figure 20: Filtered intracardiac impedance signal collected from an iLP embedded in the D.I.C.E.

The above figure provides preliminary evidence that the simulator is modulating impedance across an iLP device with respect to the system stroke volume as evidenced by an increasing in peak signal amplitude corresponding to increasing system stroke volume. In Figure 21 below, the remainder of the Z-Cardio algorithm is applied to the filtered input stream shown above to monitor changes in simulator stroke volume. The top trace scales the filtered input signal as shown previously in Figure 20. The middle trace plots the positive differential of the input signal. According to the underlying theory of the Z-Cardio algorithm, the middle trace is the rate of contraction during systole. The bottom trace plots the stroke volume estimations made by the Z-Cardio algorithm for each stroke of the simulator. 

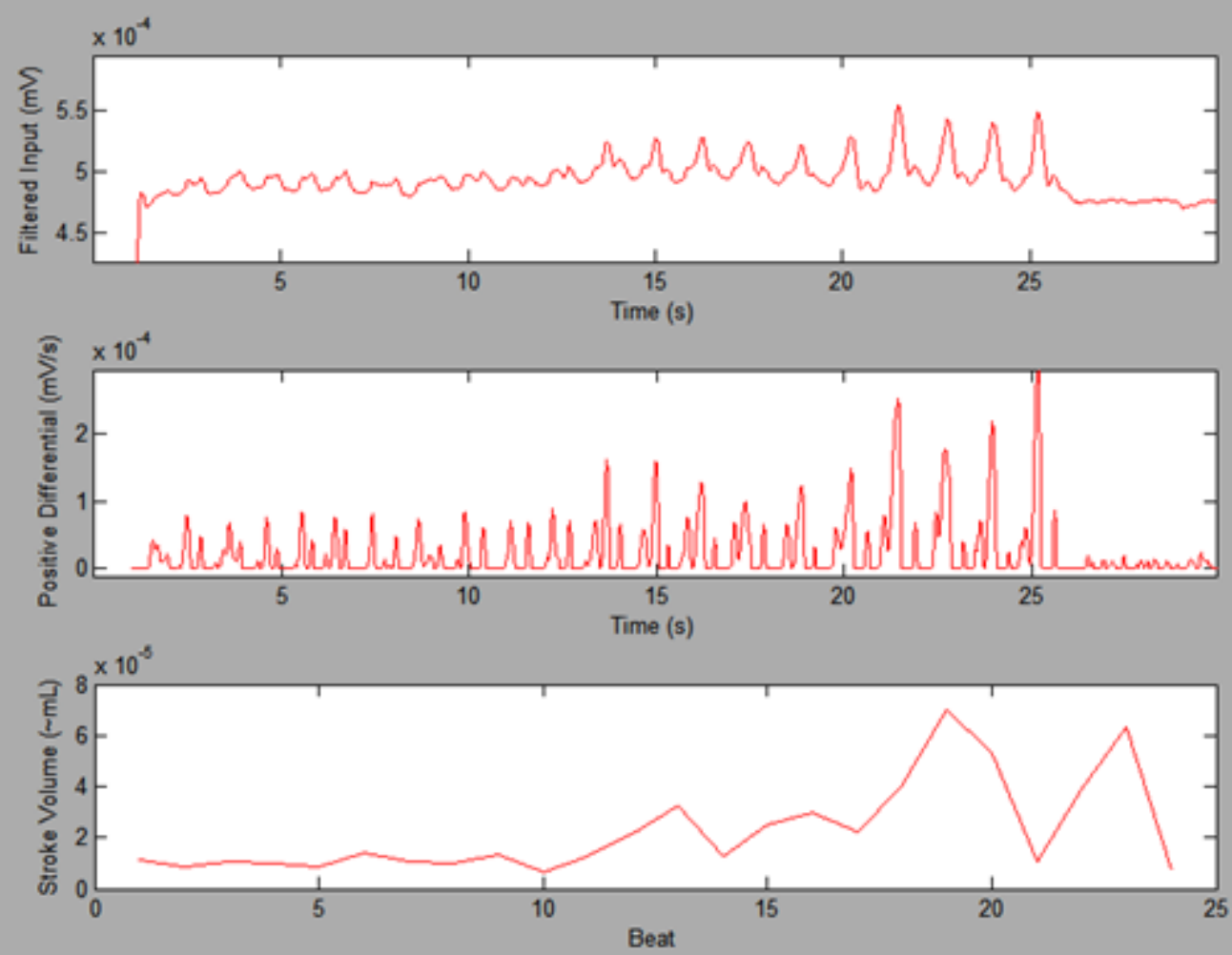

Figure 21: Illustration of the Z-Cardio algorithm signal processing cascade applied to simulated data

The three traces above in Figure 21 illustrate the relevant signal processing steps of the Z-Cardio algorithm taken to produce a stroke volume estimate. The bottom plot is the ZCardio stroke volume index, a measurement expected to be proportional to the system stroke volume. The relationship between the Z-Cardio stroke volume indices and the measured D.I.C.E. stroke volume is shown below in Figure 22. To evaluate how averaging several samples taken at the same simulated stroke volume improve the performance of the Z-Cardio algorithm, measurements were made several times at discrete intervals. 


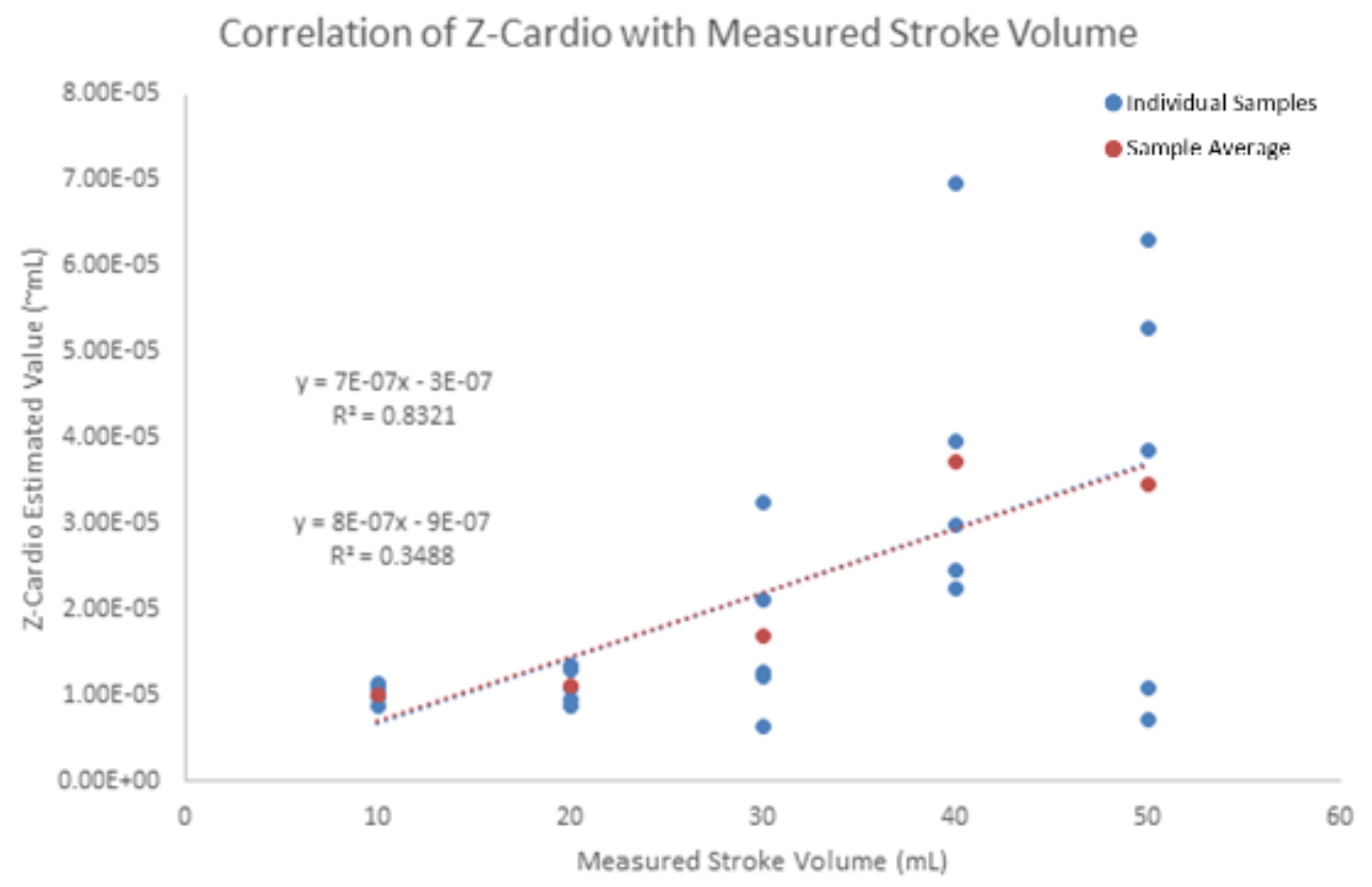

Figure 22: Linear fit between Z-Cardio stroke volume indices and the measured stroke volume of the D.I.C.E. system. The blue dots are individual measurements while the red dots are the average for the measurements made for each stroke volume

\subsection{Weaknesses}

Despite a relatively strong correlation amongst averaged samples $\left(\mathrm{R}^{2}=0.8321\right)$, significant variation exists between individual measurements for a common system stroke volume. Although the fit between all individual samples is poor $\left(R^{2}=0.3488\right)$, the fit between the average values of each stroke volume suggests a significantly stronger correlation $\left(\mathrm{R}^{2}=\right.$ 0.8321). This is an acceptable limitation because in practice a reported stroke volume estimation by Z-Cardio would be made over several contractions and not rely exclusively on individual measurements. Variation amongst individual samples may result from several factors.

First, the simulator does not control for time-varying physical dynamics, rate of 
compression, or reproducibility of impulse contraction. These features are dependent upon the human hand operating the simulator. Other factors contributing to variation could result from the geometric orientation of the device within the silicone bulb during contraction. Although it has been shown that the impedance across the device does change with stroke volume, the orientation of the device relative to the high frequency voltage generation vector would modulate the amplitude of the sensed signal. This weakness manifests itself within living subjects as well, making it a fundamental weakness of this technology. However, effects associated with cyclic vector reorientation of the iLP could be mitigated by ensuring that the vector of voltage generation is as near to the mean iLP vector as possible.

Despite various shortcoming, results obtained from the Dynamic Intra-Cardiac Emulator suggest that the Z-Cardio algorithm possesses some capacity to estimate changes in stroke volume from the intracardiac impedance signal. The simulator described in this chapter has supported demonstrating the feasibility of this technology prior to engaging in animal study. 


\section{Animal Study Design, Results, and Discussion}

Investigations focused on the features and functionality of non-approved, Class-III medical devices are not always possible in humans without first establishing their safety and efficacy in an animal model. Extensive investigations into features and functionality using in vitro and heart model simulators reduce and refine the experimental model and prototypes. Yet, present models do not completely simulate the in vivo cardiac function when integrated with the circulatory system and autonomic nervous system. The complexity of biological systems surpasses that of most bench-top simulations designed to assess a technology's performance.

Swine (Sus scrofa domestica) are an established animal model for cardiovascular device research [34]. The anatomical and physiological similarity between the human cardiovascular system and that of a swine allows the swine to accommodate necessary instrumentation while providing accurate and thorough evaluations of medical devices prior to human implantation.

Medical technology requires extensive animal evaluation before a regulatory body cedes permission to market and sell a technology to a human end user. A study protocol was developed to collect data from a living swine subject to demonstrate the feasibility of the ZCardio system. The study protocol was approved by the Institutional Review Boards (IRBs) of both Micro Systems Engineering, Inc. and Legacy Research Institute in Portland, OR. The study protocol pertinent to the Z-Cardio algorithm is available in Appendix B: In Vivo Data Collection Study Protocol.

\subsection{Outline of Study Protocol}

The purpose of the animal study was to evaluate whether the Z-Cardio algorithm is 
capable of monitoring changes in cardiac output with respect to a gold standard. A more detailed copy of the study procedures is found in Appendix C: Z-Cardio Data Collection Procedure. The following study procedures outline the steps taken within the laboratory.

1. Prepare a single swine subject for surgery, monitor vitals, induce anesthesia

2. Implant a wired iLP epoxy prototype device into the right ventricle

3. Apply surface ECG patches onto the skin for high frequency voltage generation

4. Connect a DAQ to the exposed terminal of the wired iLP

5. Insert catheters, initiate cardiac output monitoring via thermodilution (PiCCO)

6. Begin high frequency voltage generation

7. Begin recording data from the iLP through the DAQ

8. Begin streaming data from PiCCO to a USB thumb drive

9. Wait several minutes to record a baseline measurement

10. Inject a single dose of a positive inotropic pharmacological agent

11. Wait several minutes for cardiac output to spike and return to near baseline

12. Inject a second dose of a positive inotropic pharmacological agent

13. Wait several minutes for cardiac output to spike and return to near baseline

14. Inject a third dose of a positive inotropic pharmacological agent

15. Wait several minutes for cardiac output to spike and return to near baseline

16. Inject a dose of a negative inotropic pharmacological agent

17. Wait several minutes for negative inotrope to take affect

18. After several minutes, euthanize the subject per facility protocol

19. Stop recording data from both DAQ and thermodilution monitor 


\subsubsection{Gold Standard}

The purpose of this study was to evaluate whether the Z-Cardio algorithm is capable of accurately monitoring changes in cardiac output with reference to a known gold standard in a living subject. The gold standard chosen to employ in this experiment was a PiCCO (Pulse Index Continuous Cardiac Output) thermodilution cardiac output monitoring system. PiCCO was chosen because the hardware and requisite catheters were readily available, required less set-up time than other systems, provided continuous monitoring and streaming capabilities, and did not require trained professionals to operate effectively.

PiCCO monitoring integrates a wide array of both static and dynamic hemodynamic data through a combination of trans-cardiopulmonary thermodilution and pulse contour analysis [35]. The PiCCO monitor requires both venous and arterial catheters placed into the body of the subject. Iced saline is delivered through a venous catheter placed near or within

the right atrium. A thermistor catheter placed within the femoral artery monitors changes in blood temperature as the iced saline bolus passes through the vascular system. PiCCO derives an estimation of cardiac output from the contour curve of the temperature change.

While PiCCO reports true values of stroke volume and cardiac output, Z-Cardio reports indices of both stroke volume and cardiac output. This is an acceptable limitation because Z-Cardio is recognized as capable of monitoring only changes in stroke volume and cardiac output independent of the true value.

\subsubsection{Pharmacological Agents}

To assess whether the Z-Cardio algorithm is capable of monitoring changes in cardiac output, it was necessary to induce changes in cardiac output within the subject under investigation. Inotropic drugs by definition induce changes in myocardial contractility that 
modulates cardiac output. Positive inotropes increase cardiac output while negative inotropes decrease cardiac output. Dopamine, a hormone and neurotransmitter, induces vasodilation, reduces peripheral vascular resistance, and decreases vascular blood pressure. The autonomic nervous systems compensates for these effects by increasing both heart rate and myocardial contractility that contribute to an acute increase in cardiac output.

Metoprolol is a selective $\beta_{1}$ receptor blocker that blocks the action of endogenous catecholamines that mediate the fight-or-flight response in most mammals [36]. By blocking the $\beta_{1}$ receptor, nervous system stimulation is arrested, decreasing sympathetic response, heart rate, and cardiac output. Stimulating a living subject with dopamine or metoprolol will induce an increase or decrease cardiac output, respectively. Both drugs were approved for use in this study to assess whether the Z-Cardio algorithm could monitor both positive and negative changes in cardiac output.

Acute studies are short-term evaluations of device or therapy performance prior to animal sacrifice. At the conclusion of an acute study, the subject is terminated by a single bolus dose of Euthasol, a chemical barbiturate and central nervous system depressant [37]. Euthasol was approved by the institutional review boards of Micro Systems Engineering, Inc. and Legacy Research Institute as a humane means of inducing euthanasia.

\subsection{Z-Cardio Animal Study Results}

Stroke volume changes measured by both $\mathrm{PiCCO}$ and Z-Cardio are shown below in Figure 23 on the left and right, respectively. Notice three distinct sharp increases in stroke volume preceding a sharp decrease, changes that were expected as part of the study design. 


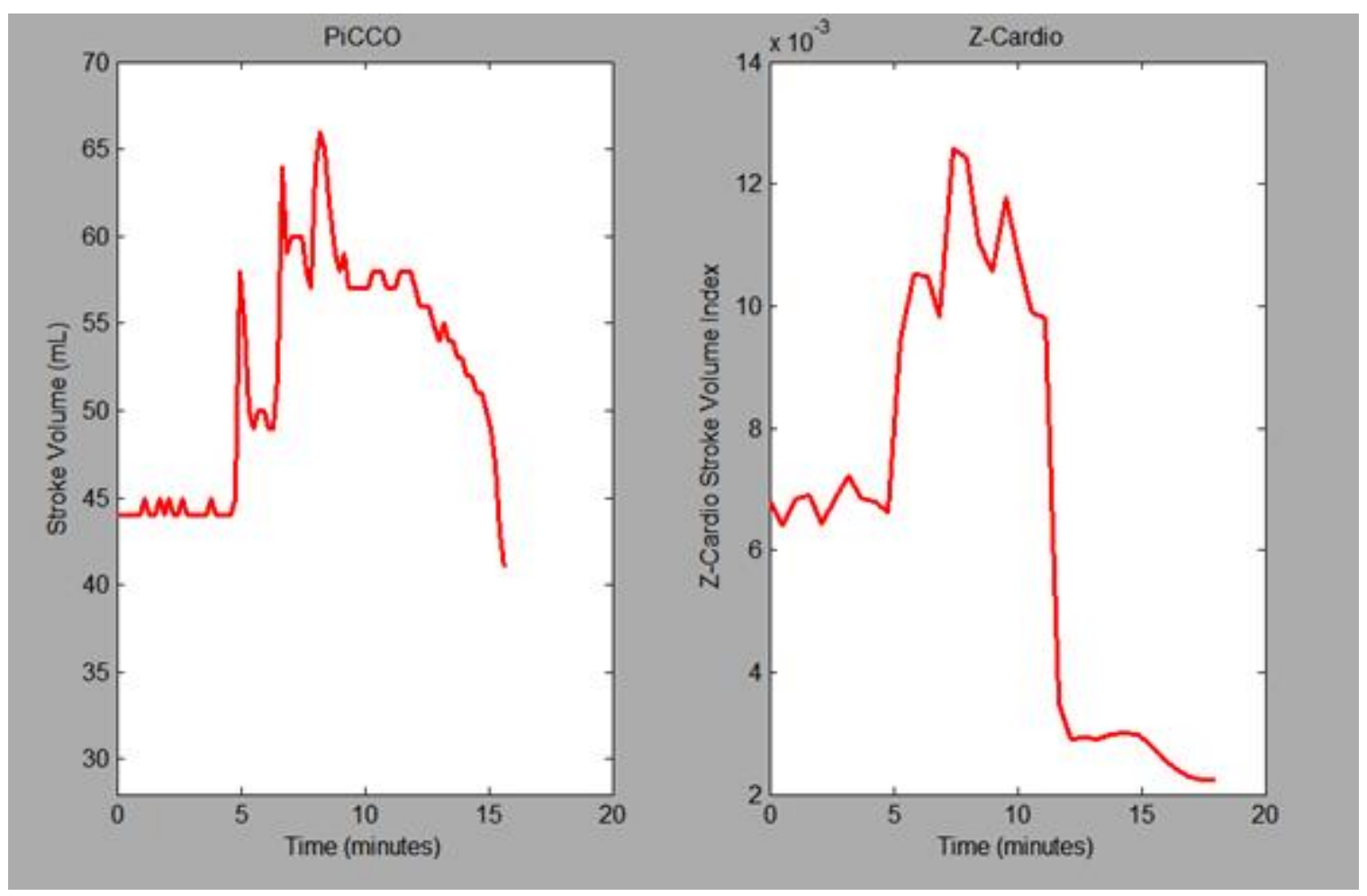

Figure 23: Stroke volume changes measured over time by both the PiCCO monitor (left) and Z-Cardio (right)

Shown above, the two estimates of stroke volume possess a high degree of similarity. In each estimate, three distinct peaks correspond to three distinct bolus doses of dopamine. The measurements above are derived from stroke volume estimations of individual cardiac contractions. Multiplied by heart rate, cardiac output estimations were derived. Cardiac output estimations made by PiCCO and Z-Cardio are shown in Figure 24 on the left and right, respectively. 


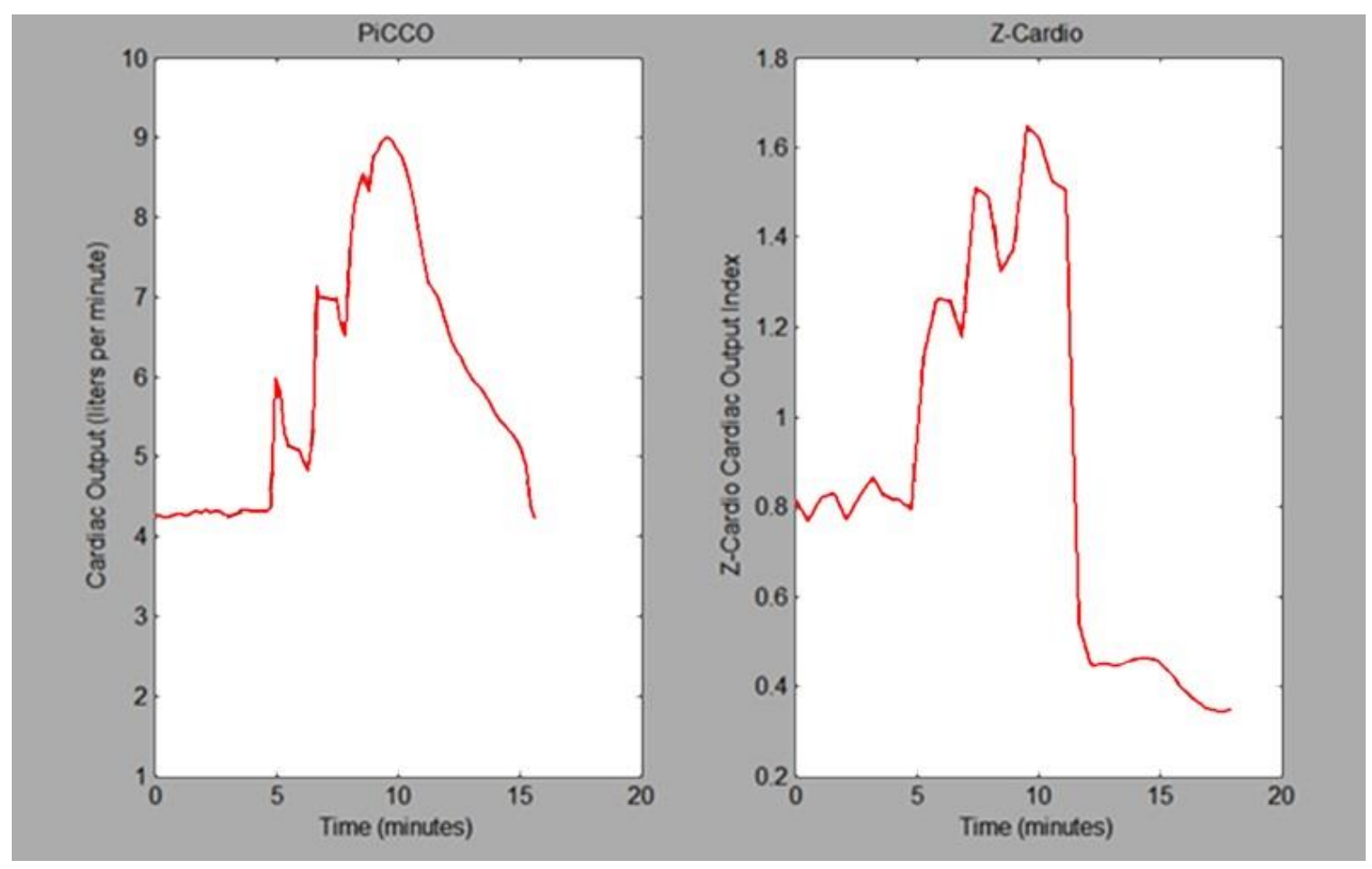

Figure 24: Cardiac output changes measured over time by both the PiCCO monitor (left) and Z-Cardio (right)

Despite the study protocol approval for the use of metoprolol to decrease cardiac output, at approximately 12 minutes following the beginning of data collection, the subject experienced a rapid decline in cardiac output not stimulated by pharmacological challenge. Under the suggestion of the attending veterinarian, the subject was euthanized prior to the administration of metoprolol. According to study protocol, the subject was euthanized with a bolus dose of Euthasol, a barbiturate that induces complete arrest of the central nervous system.

Both PiCCO and Z-Cardio again possess high degrees of similarity in estimating cardiac output. As in estimations of stroke volume, both recordings clearly illustrate three distinct spike associated with three distinct doses of dopamine. Regression analysis quantifies the relationship between the PiCCO and the Z-Cardio cardiac output estimations. The linear model between cardiac output estimations made by PiCCO and Z-Cardio is shown below in 
Figure 25.

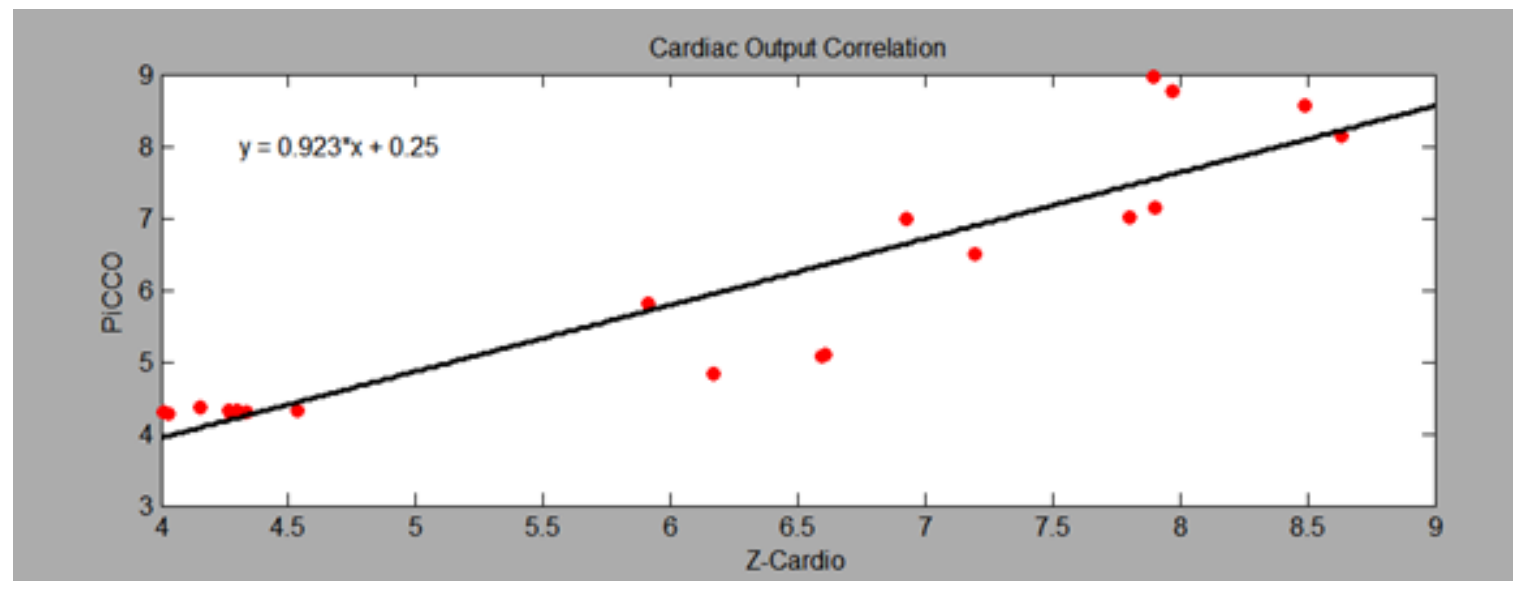

Figure 25: Regression analysis of cardiac output estimations by PiCCO and Z-Cardio

According to the linear model, the correlation coefficient between the gold standard, PiCCO, and Z-Cardio is 0.923 with an $\mathrm{R}^{2}$ value of 0.917 , indicating a moderately strong correlation between the two measurements.

\subsection{Anomalies, Weaknesses, and Interpretations}

Although promising, the results presented in this thesis are subject to anomalies and weaknesses. Most notably, the results presented here are dependent on the PiCCO thermodilution monitor as the gold standard employed for comparison. Any conclusions derived from these results are therefore dependent on the reliability of the PiCCO monitor. Studies that seek to compare cardiac output monitors should be cautious of timely calibration of thermodilution monitors

Additionally, the PiCCO monitor does not report cardiac output or stroke volume when the cardiac output index is less than $3.0 \mathrm{l} / \mathrm{min} / \mathrm{m}^{2}$, evident from the data presented. This limitation does not appear to be present in Z-Cardio; this partially accounts for discrepancies in the results but may lend credence to Z-Cardio as being an appropriate monitoring solution 
for patients with exceptionally low cardiac output who ironically are most in need of quality monitoring support. More extensive characterization is required to establish an appropriate range of measurable values for this technology.

While the study protocol was designed to induce a broad range of cardiac output in a single subject, more could be done to achieve a greater range of cardiac output changes. Ideally, changes in cardiac output could be set and held for an arbitrary duration. At present, the ideal means of controlling cardiac output to this degree would require a calibrated and programmable heart bypass machine and a massively invasive procedure. Whether feasible or not, alternative methods of inducing changes in cardiac output within a subject should be explored for future research. 


\section{Conclusions}

This thesis has described the background, hardware, theory, and algorithm necessary to monitor changes in cardiac output, as well as a bench-top intracardiac simulator used in refining the proposed algorithm and results obtained from an in vivo evaluation of the proposed cardiac output monitoring system. This thesis has employed sound engineering methodology to develop and assess a novel medical signal processing technology. However, the proposed system is far from being deployable within a human subject. The following potential avenues for further research are necessary to bring the algorithm nearer to a state sufficient for human evaluation.

\subsection{Further Research}

Further development of the Z-Cardio system and algorithm is required before the concept presented in this thesis is ready for human evaluation. The following research paths should be considered before advancing this technology beyond the proof-of-concept stage.

1. The procedures presented in this thesis could be executed while employing a more modern gold standard, such as echocardiography, in assessing how accurately the ZCardio algorithm monitors changes in cardiac output.

2. Translate the analog front-end filter into a prototype PCB, rewrite the Z-Cardio algorithm into $\mathrm{C}$ and onto an embedded chip, thereby eliminating the need for the wired iLP and extraneous hardware, such as the DAQ. Thorough design verification and validation of implantable firmware is required prior to deployment within a living subject. Additionally, requisite power consumption requirements should be characterized to ensure appropriate battery life of an implantable prototype device. 
3. Following conversion of the concept onto an embedded platform, a chronic grow-in assessment study must evaluate how well the algorithm performs over time as scar tissue and tissue capsule form around the iLP as part of the body's foreign body response.

4. Invert the concept by generating a high frequency voltage source from the iLP and monitoring changes in transthoracic impedance from outside the body, thereby offloading the signal processing requirements from the embedded iLP and onto external hardware.

5. Assess how cardiac output monitoring differs amongst individuals of different body types. While this question cannot be answered without larger scale studies, assessment of body type impact on algorithm performance will be necessary for future approval.

6. If further development of the D.I.C.E. simulator is required, consider replacing the hand pump with an electromechanical actuator for more consistent reproduction of compression dynamics.

\subsection{Concluding Remarks}

The design presented within this thesis demonstrates that an implantable device within the heart, such as a cardiac pacemaker, is capable of monitoring changes in cardiac output. Although more research is required before this type of technology is ready for chronic human implant, this promising proof of concept may pave the way for a revolution in cardiac pacing technology. An intelligent pacemaker may have the capability to automatically optimize pacing parameters by leveraging cardiac output maximization as a cost function.

Ultimately, the concept developed in this thesis is nothing more than another tool in the vast arsenal afforded to the modern physician and cardiologist. Like any tool, the value is 
dependent on the balance between the tool's strengths and weakness and how they compare to similar tools in the physician's toolbox. Launching this type of tool into the marketplace will require adherence to strict design requirements. Defining design requirements for the ZCardio algorithm will likely require substantial input from practicing clinicians, physicians, cardiologists, and nurses to better equip the design for clinical deployment. Although these requirements have not yet been investigated, substantial review of demonstrated performance would be required for most future development. Nevertheless, the groundwork presented herein may serve as a promising foundation for future medical applications and cardiac output monitoring solutions for intracardiac medical devices. 


\section{References}

[1] A. Guyton and J. Edwards, Textbook of Medical Physiology, Philadelphia: Elsevier Inc., 2006.

[2] "Medical Dictionary," MedicineNet, 2011.

[3] J. B. Hall, "Searching for Ecidence to Support Pulmonary Artery Catheter Use in Critically Ill Patients," JAMA, vol. 294, no. 13, pp. 1693-4, 2005.

[4] T. Schlosser, K. Pagonidis, C. Herborn, P. Hunold, K.-U. Waltering, T. C. Lauenstein and J. Barkhausen, "Assessment of Left Ventricular Parameters Using 16-MDCT and New Software for Endocardial and Epicardial Border Delineation," Am J Roentgenol, vol. 184, no. 3, pp. 765-73, 2005.

[5] J. Hall, Guyton and Hall Textbook of Medical Physiology (12 ed.), Philadelphia: Elsevier, Inc, 2011.

[6] P. Valensi, L. Lorgis and Y. Cottin, "Prevalence, incidence, predictive factors and prognosis of silent myocardia infarction: a review of the literature," Arch Cardiovasc Dis, vol. 104, no. 3, pp. 178-88, 2011.

[7] A. Hall and J. Guyton, Textbook of Medical Physiology (11 ed.), Philadelphia: W.B. Saunders, 2005.

[8] K. Aswini, ECG-Simplified, vol. 15, LifeHugger, 2010, pp. 408-21.

[9] R. Perez and F. Ferreira, "The enigmatic sixth wave of the electrocardiogram: the U wave," Cardiol J, vol. 15, no. 5, pp. 408-21, 2008. 
[10] G. Walraven, in Basic Arrbythmias (7th ed.), 2011, pp. 1-11.

[11] J. G. Betts, in Anatomy and Phyiosology, 2013, pp. 787-846.

[12] T. McCracken, in New Atlas of Human Anatomy, China, Metro Books, 1999, pp. $1-120$

[13] W. J. Mendel, Cardiac Arrythmias: Their Mechanisms, Diagnosis, and Management (3rd ed.), Lippincott Williams \& Wilkins, 1995.

[14] J. Custer and R. Rau, The Harriet Lane Handbook (18th ed.), Philadelphia: Elsevier, Inc, 2008.

[15] M. E. Zevitz, "Ventricular Fibrillation," Medscape.

[16] "Sinus Bradycardia," eMedicine.

[17] E. B. Fortescue, C. I. Berul, F. Cecchin, E. P. Walsh, J. K. Triedman and M. E. Alexander, "Petient, procedureal, and hardware factors associated with pacemaker lead failures in pediatrics and congenital heart disease," Heart Rhythm, vol. 1, no. 2, pp. 150-159, 2004.

[18] N. Pratini, "How much does an echocariogram cost?," 2014.

[19] K. Wesseling, J. Jansen, J. Settels and J. Schreuder, "Computation of aortic flow from pressure in humans using a nonlinear, three-element model," Journal of Applied Physiology, vol. 74, no. 5, pp. 1566-73, 1993.

[20] "Arteriovenous oxygen difference," Sports Medicine, Sports Science and Kinesiology. Net Industries, 2011.

[21] D. P. Bernstein, "Impedance Cardiography. Pulsatile blood flow and the 
biophysical and electrodynamic basis for the stroke volume equations," Journal of Electrical Bioimpedance, vol. 1, pp. 2-17, 2010.

[22] A. Sherwood, M. Allen, J. Fahrenberg, R. Kelsey, W. Lovallo and L. Van Doornen, "Methodological Guidelines for Impedance Cardiography," Psythophysiology, vol. 27, no. 1, 1990.

[23] I. Ovsyshcer and S. Furman, "Impedance Cardiography for Cardiac Output Estimation in Pacemaker Patients: Review of the Literature," Pacing and Clinical Electrophysiology, vol. 16, no. 7, pp. 1412-1422, 1993.

[24] M. Taborsky, J. Kupec, R. Volpalka, A. Barbetta and F. DiGregorio, "Left ventricular mechanical activity detected by impedance recording," Europace, vol. 12, no. 4, pp. 534-539, 2010.

[25] H. Francois, S. A. Hunt, D. N. Rosenthal and D. J. Murphy, "Right ventricular function in cardiovascular disease, part I: Anatomy, phyiology, aging, and functional assessment of the right ventricle," Circulation, vol. 117, no. 11, pp. 1436-48, 2008.

[26] R. Prakash, "Determination of right ventricular wall thickness in systole and diastole. Echocardiographic and necropsy correlation in 32 patients," Br Heart J., vol. 40, no. 11, pp. 1257-1261, 1978.

[27] R. Foale, P. Nihoyannopoulus, W. KcKenna, A. Kelinebenne, A. Nadazdin, E. Rowland, G. Smith and A. Klienebenne, "Echocardiographic measurement of the normal adult right ventricle," Br Heart J, vol. 56, pp. 33-44, 1986.

[28] R. R. Gowrishankar and J. C. Weaver, "An Approach to Electrical Modeling of 
Single and Multiple Cells," Proceedings of the National Academy of Sciences of the United States of America, vol. 100, no. 6, pp. 3203-3208, 2003.

[29] Y. Salazar, R. Bragos, O. Casas, J. Cinca and J. Rosell, "Transmural Versus Nontransmural In Situ Electrical Impedance Spectrum for Healthy, Ischemic, and Healed Myocardium," IEEE Transactions on Biomedical Engineering, vol. 51, no. 8, pp. 1421-1427, 2004.

[30] J. Ruskin, R. Bache, J. Rembert and J. Greenfield, "Pressure-Flow Studies in Man: Effect of Respiration on Left Ventricular Stroke Volume," Circulation, vol. 48, pp. 79-85, 1973.

[31] "Dielectric Properties of Body Tissue in the Frequency range $10 \mathrm{~Hz}$ to 100 GHz," Italian National Research Council, Institute for Applied Physics.

[32] S. O'Connor, Examination Medicine (The Examination), Edinburgh: Churchill Livingstone, 2009.

[33] T. Schlosser, K. Pagonidis, C. Herborn, P. Hunold, K.-U. Waltering, T. C. Lauenstein and J. Barkhausen, "Assessment of Left Ventricular Parameters Using 16-MDCT and New Software for Endocardial and Epicardial Border Delineation," Am J Roentgenol, vol. 184, no. 3, pp. 765-73, 2005.

[34] M. Swindle, A. Makin, A. Herron, F. Clubb and K. Frazier, "Swine as Models in Biomedical Research and Toxicology Testing," Veterinary Pathology, vol. 49, no. 2, pp. 344-356.

[35] E. Litton and M. Morgan, "The PiCCO Monitor: a review," Anaesth Intensive Care, vol. 40, no. 3, pp. 393-409, 2012. 
[36] W. Frishman, A. Cheng-Lai and J. Nawarskas, Current Cardiovascular Drugs, Current Science Group, 2005.

[37] DIGNITAS, 2011. 
Appendix A: D.I.C.E. Design Drawing

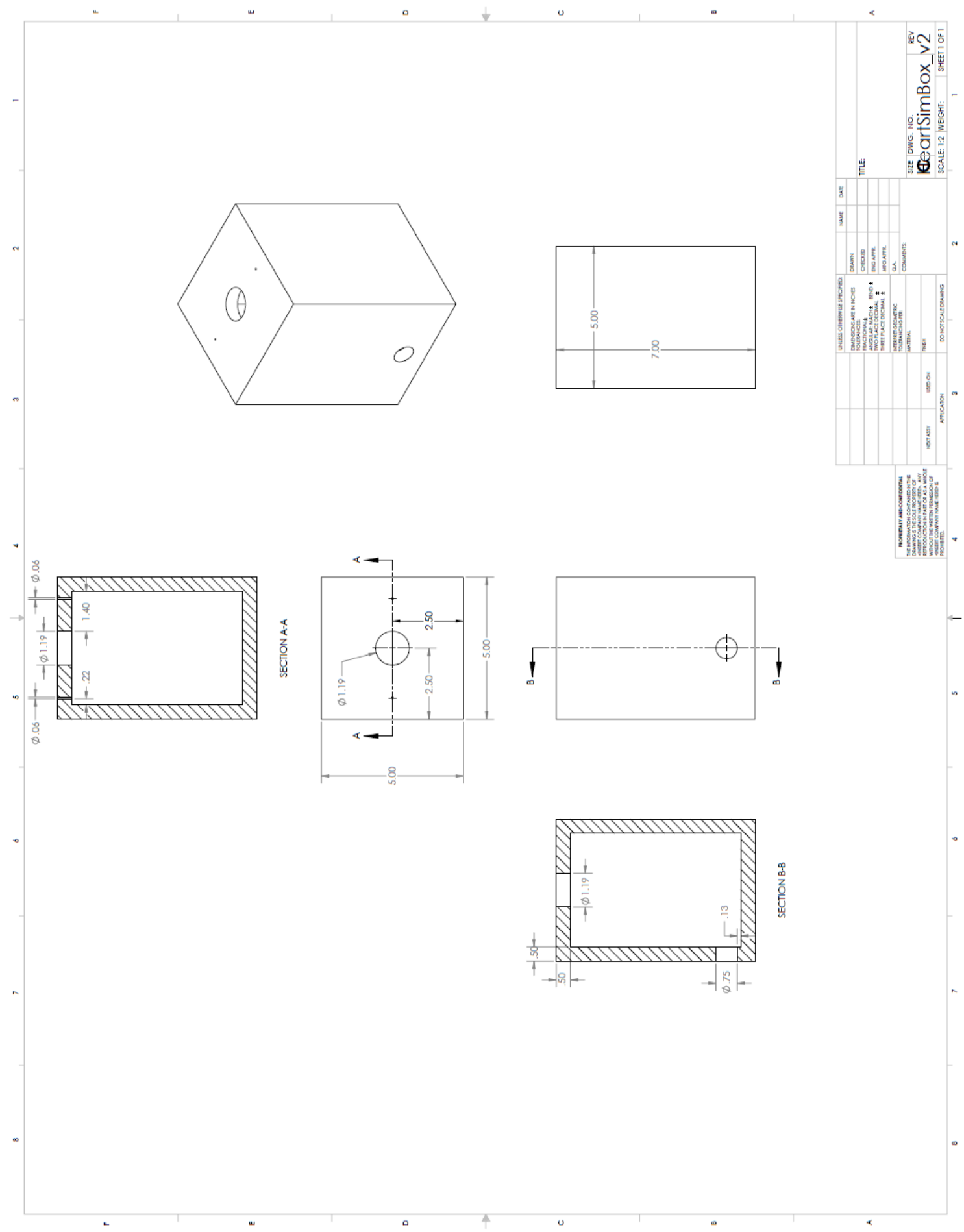




\section{Appendix B: In Vivo Data Collection Study Protocol}

The following procedures were designed to obtain data from an implanted wired iLP device while pharmacologically modulating subject stroke volume and cardiac output.. This protocol was executed as part of MSEI internal acute study ARC-2013-009 at Legacy Research Institute, Department of Comparative Medicine in Portland, Oregon. The study was an acute, non-survival study. The protocol was executed on one subject.

\section{Justification of Testing System and Animal Arrangements}

Investigations focused on the features and functionality of non-approved, Class-III medical devices are not always possible in humans without first establishing their safety and utility in an animal model. Extensive investigations into features and functionality using in vitro and heart model simulators reduce and refine the experimental model and prototypes. Yet, present models do not accurately simulate the in vivo cardiac function when integrated with the circulatory system and autonomic nervous system.

Swine (Sus scrofa domestica) is an established animal model for cardiovascular device research. The anatomical and physiological similarity between the human cardiovascular system and that of a swine allows the swine to accommodate the necessary instrumentation while providing accurate and thorough evaluations of medical devices prior to human implantation.

Prior to enrollment in the study, animals undergo medical examinations after their arrival (entrance exam) and after the acclimatization period. This process helps to ensure only healthy animals undergo experimental procedures. The breeder will provide health certificates for the animal. If during the acclimatization period a veterinarian deems medical treatment 
necessary, a member of the facility's staff will inform the sponsor immediately. The treated animal's documentation will record the type of medication/intervention, course of treatment, and reason for the intervention.

Before the animal arrives, treatment of the enclosure will conform to the facility's standard operating procedure (SOP). It is the intent of the study to house animals in a group enclosure. However, transfer of animals to a solitary enclosure may occur, as deemed necessary by the veterinarian. The room temperature will be between $21.1 \pm 2.2^{\circ} \mathrm{C}$. The relative humidity of the room is maintained between $40 \%$ and $70 \%$. Values outside these ranges may occur occasionally during extreme climatic conditions. Artificial light is set to provide a 12-14 hour light cycle, and a 10-12 hour dark cycle. The air supply is 100\% fresh (not re-circulated). Assessment of ventilation rates occur on an annual basis and following major construction. Swine pens are spot cleaned daily with complete bedding replacement weekly.

Each subject shall receive Purina Mills ${ }^{\circledR}$ Lab Porcine Grower Diet 5084. Administration of the diet (approximate amount 3-5\% of body weight per day) occurs as a split ration twice daily, and as a full ration once daily on weekends. Inspections of feedbags occur upon delivery for evidence of damage. Feed or bags found to be grossly contaminated, damaged, or repaired are not eligible for use. All food must be no more than 180 days past the milling date. Tap water is continuously available (ad libitum) from an automatic watering system until the night before the surgical procedure. On a monthly schedule, water quality undergoes a documented assessment by the Department of Comparative Medicine staff.

The subject will not have access to food or water for no less than 12 hours and no more than 18 hours before the start of the study to reduce the risk of aspiration. These times are subject to change at the veterinarian's discretion. Prior to the induction of anesthesia, the 
subject will receive a weight-adjusted subcutaneous dose of tiletamine/zolazepam and atropine as a pre-anesthetic and sedative. Transfer to the laboratory will occur once the sedatives have taken effect. In the laboratory, the subject will undergo intubation and anesthetization via IV introduction of telazol and atropine with or without carporfen or buprenorphrine. To maintain anesthesia during the experimental procedure, the subject will receive a continuous flow of oxygen mixed with $0.5-5.0 \%$ Isoflourane by volume, via the intubation tube. Inserting at least one IV line into the ear vein will allow for the administration and maintenance of fluids. Continuously warming IV fluids and providing a warming pad underneath the subject provide maintenance and regulation of the subject's body temperature. Further, a warming blanket is available as support if the subject requires additional assistance in maintaining physiologically healthy body temperatures.

Upon completion of experimental procedures, the subject will receive a barbiturate (Euthasol - $1 \mathrm{~mL} / 5 \mathrm{~kg}-\mathrm{IV})$, or another method which complies to veterinarian recommendation and IACUC (Institutional Animal Care and Use Committee) standards. Assessment of the subject's ECG, respiration, pupil dilation, and cardiac activity will confirm euthanasia prior to the initiation of the necropsy. Following the conclusion of testing, an explantation procedure for the heart will begin. The explanted material may include the following:

○ The Heart

- $3 \pm 3 \mathrm{~cm}$ of Inferior Vena Cava

- $3 \pm 3 \mathrm{~cm}$ of Superior Vena Cava

○ $3 \pm 3 \mathrm{~cm}$ of Pulmonary Artery

○ $3 \pm 3 \mathrm{~cm}$ of Pulmonary Vein 


\section{$\circ \quad 3 \pm 3 \mathrm{~cm}$ of Aorta}

Upon explant, the heart will undergo dissection and examination. Visual observations, notes, and photographs will record the implantation procedure and tool removal. If necessary, tissue samples will be collected and transported back to Biotronik, Inc./Micro Systems Engineering, Inc. in an isotonic saline bath or in formalin or sent to a histo-pathologist for analysis.

Throughout the study, pharmacological support may be administered to support the subject's vitals or to achieve a desired physiological effect. Table 2 lists drugs available to support the subject as needed. The name, pharmacological family, quantity available, dosage, anticipated duration of effect, and the desired effect of each drug is listed in the table.

Table 2: Pharmacological drugs administered as part of this study

\begin{tabular}{|c|c|c|c|c|c|}
\hline Name & Family & Quantity & Dose & $\begin{array}{l}\text { Duration } \\
\text { of Effect }\end{array}$ & Desired Effect \\
\hline Dopamine & $\begin{array}{l}\text { Sympathomimetic } \\
\text { Agonist }\end{array}$ & 3 Vials & $\begin{array}{l}2 \text { to } 20 \mu \mathrm{g} / \mathrm{kg} / \mathrm{min} \\
\mathrm{IV} \text { push doses } 1 \mathrm{mg} \text {, } \\
2 \mathrm{mg}, 3 \mathrm{mg}(6 \mathrm{mg} \max ) \text { to } \\
\text { CVP } \\
\text { Vasopressive dose } 15 \text { to } \\
50 \mathrm{ug} / \mathrm{kg} / \mathrm{min}\end{array}$ & $<10$ Mins & $\begin{array}{l}\text { Positive } \\
\text { Chronotrope } \\
\text { Positive Inotrope }\end{array}$ \\
\hline Metoprolol & $\begin{array}{l}\text { Sympathomimetic } \\
\text { Antagonist ( } \beta- \\
\text { blocker) }\end{array}$ & $120 \mathrm{mg}$ & $\begin{array}{l}1 \text { to } 5 \mathrm{mg} / \mathrm{min} \\
(7 \mathrm{minute} \max .) \\
\text { IV push doses } 5 \mathrm{mg} \\
10 \mathrm{mg}, 10 \mathrm{mg}(25 \mathrm{mg} \\
\max ) \text { to CVP }\end{array}$ & $\begin{array}{l}>120 \\
\text { Mins }\end{array}$ & $\begin{array}{l}\text { Negative } \\
\text { Chronotrope } \\
\text { Negative Intrope }\end{array}$ \\
\hline
\end{tabular}

Justification of Test Hardware Design

Surface electrodes will transmit a voltage at frequency between 100 and $400 \mathrm{kHz}$ across the thorax while the subject's stroke volume is pharmacologically modulated. Raw data will be streamed continuously to disk by a National Instruments data acquisition system (NIDAQ) USB-6343 through MATLAB. The NIDAQ will connect to both proximal ring and tip of the wired iLP. The National Instruments system will record data at between 100 and $500 \mathrm{kHz}$ at 
16 bit resolution. The data file will be stored to and saved directly onto the laptop's hard drive.

A wired iLP device possesses two electrodes at both the tip and the hitch. The tip and hitch are connected to the terminal by a pair of 50 centimeter drawn filled tube (DFT) wires. The proximal ends of the wires connect to a standard IS-1 pacemaker lead terminal. Measurements are recorded from the tip (the positive terminal at the iLP screw) and the ring (the negative terminal at the iLP fin) of the terminal. Measuring voltage across the terminal leads is analogous to measuring voltage across the iLP.

\section{Equipment}

The data collection study will employ specific hardware and software to collect and store the data obtained from this study.

Table 3: Quantity and equipment employed throughout the preliminary data collection study

\begin{tabular}{ll}
\hline Quantity & Device \\
\hline 1 & Laptop with Windows 7 running MATLAB \\
1 & National Instruments USB-6343 Data Acquisition System \\
1 & Pulsion Medical Systems PiCCO ${ }_{2}$ Monitor with Cables and Catheters \\
1 & Isolated Z-COMM Signal Transmitter Board \\
2 & Silver chloride Surface Electrodes \\
1 & Extension Cord \\
1 & Power Strip \\
1 & External Hard Drive (500 GB) \\
1 & Wired iLP Device \\
1 & iLP Deployment Tool \\
\hline
\end{tabular}




\section{Appendix C: Z-Cardio Data Collection Procedure}

1. Prepare $500 \mathrm{~mL}$ cold saline and keep on ice

2. Shave the subject's chest and clean the skin with a diluted isopropyl alcohol solution

3. Perform bilateral jugular cut downs at the left and right jugular furrow

4. Isolate both the left and right external jugular veins, ligate the vessels, pack incision with moistened gauze as needed

5. Implant a wired iLP device per pre-defined iLP implantation procedure

6. Using fluoroscopy, identify the position and orientation of the iLP within the heart

7. Mark the skin surface with a marker on both coronal and sagittal planes

8. Assemble the PiCCO cardiac output monitoring system

9. Implant PiCCO thermodilution catheters per pre-defined PiCCO setup procedure

10. Once catheterized, calibrate the PiCCO monitor via administration of three cold saline boluses

11. Place two transmitting ECG surface electrodes on the subject's chest axially aligned with the iLP device. The electrodes should be separated by approximately twice the depth of the iLP from the skin surface

12. Connect the terminal ends of the wired iLP to the NIDAQ

13. Connect the transmitting electrodes to an isolated battery-powered Z-COMM function-generating programmer board

14. Set the sinusoidal transmission frequency to $100 \mathrm{kHz}$ at amplitude such that measured voltage amplitude at the implant device is approximately $15 \mathrm{mV}$.

15. Simultaneously begin streaming data from both the PiCCO monitor and the NIDAQ 
via MATLAB

16. Wait approximately 1 minute to collect baseline data

17. Administer IV push doses $1 \mathrm{mg}$ of dopamine to CVP to increase subject inotropy.

a. Observe any changes in heart rate, strove volume, or cardiac output

b. Wait for cardiac output to return to near baseline or 5 minutes, whichever comes first

18. Administer IV push doses $2 \mathrm{mg}$ of dopamine to CVP to increase subject inotropy.

a. Observe any changes in heart rate, strove volume, or cardiac output

b. Wait for cardiac output to return to near baseline or 5 minutes, whichever comes first

19. Administer IV push doses $3 \mathrm{mg}$ of dopamine to CVP to increase subject inotropy.

a. Observe any changes in heart rate, strove volume, or cardiac output

b. Wait for cardiac output to return to near baseline or 5 minutes, whichever comes first

20. Following the maximum dose of dopamine, allow the vital signs to approach baseline values

21. Administer IV push doses $25 \mathrm{mg} \max$ to CVP of metoprolol to reduce subject inotropy.

22. Once vital signs have stabilized under metoprolol, or after 5 minutes, administer 1 $\mathrm{mL} / 5 \mathrm{~kg}$ of Euthasol to terminate the subject.

23. Once the animal is declared deceased, stop recording from both PiCCO and NIDAQ 